Pacific

Journal of

Mathematics

ON SOME VARIANTS OF THE KAKEYA PROBLEM

LaWrence Kolasa and Thomas WolfF 


\title{
ON SOME VARIANTS OF THE KAKEYA PROBLEM
}

\author{
LaWrence Kolasa and Thomas WolfF
}

\begin{abstract}
We study the question of lower bounds for the Hausdorff dimension of a set in $R^{n}$ containing spheres of every radius. If $n \geq 3$ then such a set must have dimension $n$. If $n=2$ then it must have dimension at least 11/6. We also study the analogous maximal function problem and related problem of Besicovitch sets with an axis of symmetry.
\end{abstract}

Besicovitch and Rado [1] and Kinney [5] proved the following result: There is a closed set $E \subset \mathbb{R}^{2}$ with measure zero which contains a circle of every radius.

The construction of Besicovitch and Rado works in $\mathbb{R}^{d}$ : If $d \geq 3$ there is a closed set $E \subset \mathbb{R}^{d}$ with measure zero which contains a sphere of every radius. We will give an exposition of this construction in Section 1 below.

One can ask whether a set containing a sphere of every radius must have Hausdorff dimension $d$. As it turns out, this question is easily answered in higher dimensions.

Theorem 1. A Borel set in $\mathbb{R}^{d}, d \geq 3$ which contains a sphere of every radius has Hausdorff dimension $d$.

In $\mathbb{R}^{2}$ this may still be true but appears harder. One purpose of this paper is to prove the following partial result.

Theorem 2. A Borel set in $\mathbb{R}^{2}$ which contains a circle of every radius has Hausdorff dimension at least $\frac{11}{6}$.

Following a known pattern (see [2] for example) we will derive Theorems 1 and 2 from $L^{p} \rightarrow L^{q}$ estimates for a related maximal function. Fix $\delta>0$. For $x \in \mathbb{R}^{d}, r \in\left[\frac{1}{2}, 2\right]$, define

$$
\begin{aligned}
C(x, r) & =\left\{z \in \mathbb{R}^{d}:|z-x|=r\right\} \\
C_{\delta}(x, r) & =\left\{z \in \mathbb{R}^{d}: r-\delta<|z-x|<r+\delta\right\} .
\end{aligned}
$$

If $f: \mathbb{R}^{d} \rightarrow \mathbb{R}$ then define $M_{\delta} f:[1 / 2,2] \rightarrow \mathbb{R}$ via

$$
M_{\delta} f(r)=\sup _{x} \frac{1}{\left|C_{\delta}(x, r)\right|} \int_{C_{\delta}(x, r)}|f|,
$$

where $|E|$ means Lebesgue measure of $E$. In higher dimensions we have the following estimate: 
Theorem 1'. $\left\|M_{\delta} f\right\|_{L^{2}([1 / 2,2])} \leq C_{d}\left(\log \frac{1}{\delta}\right)^{1 / 2}\|f\|_{2}, d \geq 3$. In fact, if $d \geq 3$ there are $L^{p} \rightarrow L^{q}$ estimates

$$
\left\|M_{\delta} f\right\|_{L^{q}([1 / 2,2])} \leq C\left(\log \frac{1}{\delta}\right)^{1 / p}\|f\|_{p}
$$

if $p \geq 2$ and $q \leq p$ and

$$
\left\|M_{\delta} f\right\|_{L^{q}([1 / 2,2])} \leq C \delta^{-\alpha}\|f\|_{p}
$$

for other $p, q$, where $\alpha=\max \left(\frac{1}{p}-\frac{1}{p^{\prime}}, \frac{1}{p}-\frac{1}{q}\right)$.

Theorem $1^{\prime}$ follows easily from (for example) the method of A. Cordoba [3] and we expect that it may have been observed before, although we don't know a reference and will therefore give a proof, in Section 2.

Theorem $1^{\prime}$ is easily seen to fail in in $\mathbb{R}^{2}$, as we will show in Section 3 . The correct question appears to be whether there is an estimate

$$
\forall \epsilon>0 \exists C_{\epsilon}:\left\|M_{\delta} f\right\|_{L^{3}([1 / 2,2])} \leq C_{\epsilon} \delta^{-\epsilon}\|f\|_{3}, \quad d=2 .
$$

By interpolation with the trivial estimate $\left\|M_{\delta} f\right\|_{\infty} \leq C \delta^{-1}\|f\|_{1}$, an equivalent question is whether there is an estimate

$$
\forall \epsilon>0 \exists C_{\epsilon}:\left\|M_{\delta} f\right\|_{L^{q}([1 / 2,2])} \leq C_{\epsilon} \delta^{-\frac{1}{2}\left(\frac{3}{p}-1\right)-\epsilon}\|f\|_{p}, \quad p \leq 3, q \leq 2 p^{\prime}, d=2 .
$$

When $p=2$ (hence $q=4$ ) this estimate is due to Pecher [9] (Theorem 1, the case $n=2$ and $q=\infty$ ), in an equivalent form referring to solutions of the wave equation, but non- $L^{2}$ estimates appear to require different ideas.

We will prove (4) when $p \leq \frac{8}{3}$, using a geometric approach related to that of Marstrand [7]. Actually, we will prove (as does Pecher when $p=2$ ) the following slightly stronger "endpoint" result.

Theorem $2^{\prime}$. If $d=2, p<8 / 3, q \leq 2 p^{\prime}$ then

$$
\left\|M_{\delta} f\right\|_{q} \leq C \delta^{-\frac{1}{2}\left(\frac{3}{p}-1\right)}\|f\|_{p}
$$

Whether (4) holds when $8 / 3<p \leq 3$, and whether a set in $\mathbb{R}^{2}$ containing a circle of every radius has Hausdorff dimension 2, at present are open problems. These problems can be regarded as special cases of the local smoothing problem for the wave equation [10], [8] - a slightly weaker form of (3) (namely: The same estimate with the $L^{3}$ norm replaced by the $L^{4}$ norm on both sides of the inequality) would follow readily from the "sharp local smoothing" conjecture made in [10], [8].

Another closely related problem, which was the original motivation for this paper, is the three dimensional Kakeya problem specialized to the cylindrically symmetric case. More precisely define a rotation invariant function 
to be a function $f: \mathbb{R}^{3} \rightarrow \mathbb{R}$ such that

$$
\begin{aligned}
x=\left(x_{1}, x_{2}, x_{3}\right) \in & \mathbb{R}^{3}, \theta \in \mathbb{R} \\
& \Rightarrow f\left(x_{1} \cos \theta+x_{2} \sin \theta,-x_{2} \cos \theta+x_{1} \sin \theta, x_{3}\right)=f(x)
\end{aligned}
$$

and a rotation invariant set to be a set whose characteristic function is rotation invariant. A Besicovitch set is a Borel set in $\mathbb{R}^{3}$ containing a line segment in every direction. We will prove:

Theorem 3. Any rotation invariant Besicovitch set has Hausdorff dimension at least $\frac{17}{6}$.

For general sets, the best bound presently known is $\frac{5}{2}$, proved in $[\mathbf{1 3}]$ which contains references to relevant previous work.

Bourgain [2] realized that the maximal function most closely related to the Kakeya problem is the following $f_{\delta}^{*}$. If $f: \mathbb{R}^{3} \rightarrow \mathbb{R}, \delta>0, \mathbb{P}^{2}$ is two dimensional projective space then one defines $f_{\delta}^{*}: \mathbb{P}^{2} \rightarrow \mathbb{R}$ by

$$
f_{\delta}^{*}(e)=\sup _{T_{e}^{\delta}} \frac{1}{\left|T_{e}^{\delta}\right|} \int_{T_{e}^{\delta}}|f|
$$

where $T_{e}^{\delta}$ runs over all cylinders with length 1 , cross section radius $\delta$ and axis in the $e$ direction. We will prove the following.

Theorem $3^{\prime}$. If $f: \mathbb{R}^{3} \rightarrow \mathbb{R}$ is rotation invariant then

$$
\left\|f_{\delta}^{*}\right\|_{L^{q\left(\mathbb{P}^{2}\right)}} \leq C \delta^{-\left(\frac{3}{p}-1\right)}\|f\|_{p}, \quad p<\frac{17}{6}, \quad q \leq 2 p^{\prime} .
$$

To see why Theorems $2^{\prime}$ and $3^{\prime}$ are related, note first that although Theorem $3^{\prime}$ is formulated in $\mathbb{R}^{3}$, it is really a two dimensional result because of the rotation invariance. We will show in Section 5 that it basically reduces to a result like Theorem $2^{\prime}$ with hyperbolas instead of circles. Namely, if $\delta>0$ is fixed and if $x, y, z$ are real numbers with $x>0$ and $\frac{1}{2} \leq z \leq 2$ then we define

$$
\begin{aligned}
& \text { (5) } H(x, y, z)=\left\{(s, t) \in \mathbb{R}^{2}: s>0, s^{2}-\frac{(t-y)^{2}}{z}=x\right\} \\
& \text { (6) } H_{\delta}(x, y, z)=\left\{(s, t) \in \mathbb{R}^{2}: \frac{1}{2} \leq s \leq 2, \operatorname{dist}((s, t), H(x, y, z))<\delta\right\},
\end{aligned}
$$

and for $f: \mathbb{R}^{2} \rightarrow \mathbb{R}$ we define

$$
\tilde{M}_{\delta} f(z)=\sup _{x>0, y \in \mathbb{R}} \frac{1}{\delta} \int_{H_{\delta}(x, y, z)}|f| .
$$

Lemma 5.1 below is the result analogous to Theorem $2^{\prime}$ for $\tilde{M}_{\delta}$. In fact, we will obtain both Theorem $2^{\prime}$ and Lemma 5.1 as special cases of a more 
general result (Theorem 4.1) for families of curves satisfying the "cinematic curvature" condition from $[\mathbf{1 0}]$.

Theorem $3^{\prime}$ bears the same relation to Theorem 3 as Theorem $2^{\prime}$ does to Theorem 2. In fact, the implication (Theorem $3^{\prime} \Rightarrow$ Theorem 3 ) follows by specializing the argument in [2], Lemma 2.15 to the cylindrically symmetric case, while the following lemma shows that Theorems $1^{\prime}$ and $2^{\prime}$ imply Theorems 1 and 2 .

Lemma 0.1. An estimate in $\mathbb{R}^{d}$ of the form

$$
\left\|M_{\delta} f\right\|_{q} \leq C \delta^{-\alpha}\|f\|_{p}
$$

implies that a Borel set $E \subset \mathbb{R}^{d}$ containing a positive $(d-1$ dimensional $)$ measure subset of a sphere of radius $r$ for a positive measure set of $r$ has Hausdorff dimension at least $d-p \alpha$.

The proof of this is identical to the proof of Lemma 2.15 in [2], so we omit it.

We use the notation $x \lesssim y$ to mean that $x \leq C y$ for a suitable fixed constant $C$, and similarly with $x \gtrsim y$ and $x \approx y$. The rest of the paper is organized as follows: In Section 1 we carry out the Besicovitch-Rado construction in general dimensions and in Section 2 we prove Theorem 1'. In Section 3 we extend lemmas from [7] to the setting of curves with cinematic curvature, and in Section 4 we prove Theorem $2^{\prime}$ and the above mentioned generalization. The argument in Section 4 is the main point of the paper. In Section 5 we prove Theorem $3^{\prime}$.

Acknowledgments. We are indebted to P. Mattila who pointed out the similarity between our arguments and Marstrand's. The fact that the $p=2$ case of Theorem $2^{\prime}$ is in the wave equation literature was pointed out by $\mathrm{C}$. Sogge and by C. Kenig and L. Vega.

Added 5/15/97. This paper was first written in the fall of 1994 and underwent several revisions as the authors became aware of relevant previous references, etc. Since that time several further related results have been proved by the authors and by W. Schlag. In particular, the conjecture (3) has been proved by the second author in "A Kakeya-type problem for circles" (to appear in Amer. J. Math), using extensions of the techniques of the present paper.

\section{The Besicovitch-Rado construction.}

In this section we generalize the Besicovitch-Rado-Kinney result to $\mathbb{R}^{d}$.

Proposition 1.1. For any $d \geq 2$ there is a closed subset $E \subset \mathbb{R}^{d}$, with Lebesgue measure zero which contains a sphere of every radius. 
We will follow the idea of Besicovitch and Rado, i.e., will set up a recursive Perron tree type construction where the sets to be translated are annuli rather than triangles. The Besicovitch-Rado construction can in fact be generalized to higher dimensions with essentially no changes. However, we will give a slightly different construction instead. This leads to a better estimate (at least in low dimensions) in Proposition 2.2 below.

We need a certain geometric fact, Lemma 1.1 below. In $\mathbb{R}^{d}$ consider two spheres $S_{1}$ and $S_{0}$ of radii $P_{1}$ and $P_{0}$, where $P_{1}>P_{0}$. Suppose these spheres have centers $C_{1}$ and $C_{0}$ respectively such that $\left|C_{1}-C_{0}\right|<P_{1}-P_{0}$, and let $\alpha=P_{1}-P_{0}-\left|C_{1}-C_{0}\right|$. Consider a ray, $R$, which emanates from $C_{1}$, and let $\theta$ be the angle between $R$ and the ray through the two centers $C_{1}$ and $C_{0}$ which also originates at $C_{1}$.

Lemma 1.1. The length of the segment of $R$ between $S_{0}$ and $S_{1}$ is

$$
\approx \alpha+\frac{1}{2} \theta^{2}\left|C_{1}-C_{0}\right|\left(1+\frac{\left|C_{1}-C_{0}\right|}{P_{0}}\right),
$$

when $\theta \frac{\left|C_{1}-C_{0}\right|}{P_{0}} \ll 1$ and $\theta \ll 1$.

Proof. Let $x$ denote the portion of $R$ between $C_{1}$ and $S_{0}$. What we wish to calculate is $P_{1}-|x|$. By the law of cosines $P_{0}^{2}=\left|C_{1}-C_{0}\right|^{2}+|x|^{2}-2|x| \mid C_{1}-$ $C_{0} \mid \cos \theta$ and therefore

$$
|x|=\left|C_{1}-C_{0}\right| \cos \theta+P_{0} \sqrt{1-\frac{\left|C_{1}-C_{0}\right|^{2}}{P_{0}^{2}} \sin ^{2} \theta} .
$$

Given that $\theta \frac{\left|C_{1}-C_{0}\right|}{P_{0}} \ll 1$ and $\theta \ll 1$ are small,

$$
\begin{aligned}
P_{1}-|x|= & P_{1}-P_{0} \sqrt{1-\frac{\left|C_{1}-C_{0}\right|^{2}}{P_{0}^{2}} \sin ^{2} \theta}-\left|C_{1}-C_{0}\right| \\
& +\left|C_{1}-C_{0}\right|(1-\cos \theta) \\
\geq & P_{1}-P_{0}-\left|C_{1}-C_{0}\right|+\frac{1}{2} \theta^{2}\left|C_{1}-C_{0}\right|\left(\left(\frac{\left|C_{1}-C_{0}\right|}{P_{0}}\right) \frac{\sin ^{2} \theta}{\theta^{2}}\right. \\
& \left.+2 \frac{1-\cos \theta}{\theta^{2}}\right) \\
\approx & \alpha+\frac{1}{2} \theta^{2}\left|C_{1}-C_{0}\right|\left(\left(\frac{\left|C_{1}-C_{0}\right|}{P_{0}}\right)+1\right) .
\end{aligned}
$$

The inequality on the second line holds since $\sqrt{1-x} \leq 1-\frac{1}{2} x$ for small positive $x$. The reverse inequality also holds provided the constant $\frac{1}{2}$ is replaced by a slightly larger one and $\theta \frac{\left|C_{1}-C_{0}\right|}{P_{0}}$ is small. This finishes the proof of the lemma. 
The typical step in the recursive process is as follows. Let $A$ be an annulus in $\mathbb{R}^{d}$ with center $x_{0}, A=\left\{x \in \mathbb{R}^{d}: P_{0} \leq\left|x-x_{0}\right| \leq P_{1}\right\}$, and call $P_{1}-P_{0}$ the width of $A$. We assume that $1 / 2 \leq P_{0}<P_{1} \leq 2 P_{0} \leq 2$ throughout; this serves to make all constants independent of $P_{0}$ and $P_{1}$. For fixed $n$ and fixed $e \in S^{d-1}$ we describe $\phi_{e}^{n}(A)$. First divide $A$ into $n$ concentric annuli (each of width $\left.\left(P_{1}-P_{0}\right) / n\right)$, and translate them in the $e$-direction relative to the center, $x_{0}$, of $A$ as so: Numbering the annuli form 1 to $n$ in order of largest to smallest, the $k$-th annulus is translated a distance $(k-1)\left(P_{1}-P_{0}\right) / n$. In this way the $n$ new annuli intersect tangentially ${ }^{1}$ along a ray in the $e$-direction; we call $\phi_{e}^{n}(A)$ a figure and $x_{0}$ the center of this figure.

Clearly the measure of a figure is smaller than that of the original annulus. Let $N_{\delta}(E)=\{x: \operatorname{dist}(x, E)<\delta\}$ - the $\delta$-neighborhood of $E$ - and let $\Gamma_{\theta}^{e}\left(x_{0}\right)$ denote the cone based at $x_{0}$ of width $\theta$ in the direction of $e-$ i.e.,

$$
\Gamma_{\theta}^{e}\left(x_{0}\right)=\left\{x_{0}+t h: t \geq 0, h \in S^{d-1}, \operatorname{dist}(h, e) \leq \theta\right\},
$$

where distance is measured along $S^{d-1}$. Note that if $x_{0}$ is the center of $\phi_{e}^{n}(A)$ and if $c_{0}$ is the center of its smallest annulus, then

$$
\begin{aligned}
\phi_{e}^{n}(A) \cap \Gamma_{\theta}^{e}\left(x_{0}\right) \subset \Gamma_{\theta}^{e}\left(x_{0}\right) \cap & \\
& \left\{x \in \mathbb{R}^{d}:\left|x-x_{0}\right| \leq P_{1}\right\} \cap\left\{x \in \mathbb{R}^{d}:\left|x-c_{0}\right| \geq P_{0}\right\} .
\end{aligned}
$$

Lemma 1.2. Let $A, n$, e be as above. Then for (absolutely) large $n$,

$$
\left|N_{2\left(P_{1}-P_{0}\right) / n}\left(\phi_{e}^{n}(A) \cap \Gamma_{2 / \sqrt{n}}^{e}\left(x_{0}\right)\right)\right| \lesssim\left(P_{1}-P_{0}\right) n^{-\frac{d+1}{2}} .
$$

Proof. We calculate volume using polar coordinates. Consider any ray in $\Gamma_{2 / \sqrt{n}}^{e}\left(x_{0}\right)$; we claim its intersection with $\phi_{e}^{n}(A)$ is contained in a segment of length $\approx\left(P_{1}-P_{0}\right) / n$. Indeed by Lemma 1.1 with $\alpha=\left(P_{1}-P_{0}\right) / n$ and $\left|C_{1}-C_{0}\right|=\left(P_{1}-P_{0}\right) \frac{n-1}{n}$, taking $\theta=2 / \sqrt{n}$ we see that such a segment has length $\approx \alpha=\left(P_{1}-P_{0}\right) / n$. Then $\phi_{e}^{n}(A) \cap \Gamma_{2 / \sqrt{n}}^{e}\left(x_{0}\right)$ is contained in a rectangle which has dimensions $\approx\left(P_{1}-P_{0}\right) \cdot n^{-1} \underbrace{\times n^{-1 / 2} \times \cdots \times n^{-1 / 2}}_{d-1 \text { times }}$, and hence so is $N_{2\left(P_{1}-P_{0}\right) / n}\left(\phi_{e}^{n}(A) \cap \Gamma_{2 / \sqrt{n}}^{e}\left(x_{0}\right)\right)$.

Our construction involves a repeated application of $\phi_{n}^{e}$ where we vary the direction, $e$, of translation. Let us therefore cover $S^{d-1}$ with a maximal $\frac{1}{C \sqrt{n}}$-separated subset $D=\left\{e_{1}, e_{2}, \ldots, e_{M}\right\}$, where $M \approx n^{\frac{d-1}{2}}$. Here $C$ is a sufficiently large constant. If $A=\bigcup A_{j}$ is the union of non-concentric annuli $A_{j}$, let $\phi_{e}^{n}(A)=\bigcup \phi_{n}^{e}\left(A_{j}\right)$.

Lemma 1.3. Let $A=\left\{x \in \mathbb{R}^{d}: P_{0} \leq\left|x-x_{0}\right| \leq P_{1}\right\}$, and consider points $e_{k}, e_{k+1}, \ldots, e_{k+j} \in D$. Then for (absolutely) large $n$,

\footnotetext{
${ }^{1}$ Besicovitch and Rado use a slightly different translation rule and obtain transverse intersections.
} 


$$
\begin{aligned}
\phi_{e_{k+j}}^{n} \circ \cdots \circ \phi_{e_{k+1}}^{n} \circ \phi_{e_{k}}^{n}(A) & \cap \\
& \Gamma_{2 / \sqrt{n}}^{e_{k}}\left(x_{0}\right) \subset N_{2\left(P_{1}-P_{0}\right) / n}\left(\phi_{e_{k}}^{n}(A) \cap \Gamma_{2 / \sqrt{n}}^{e_{k}}\left(x_{0}\right)\right) .
\end{aligned}
$$

Proof. By construction, a point of $\phi_{e_{k+j}}^{n} \circ \cdots \circ \phi_{e_{k+1}}^{n} \circ \phi_{e_{k}}^{n}(A)$ has been translated a distance no more than $\left(P_{1}-P_{0}\right)\left(\frac{1}{n}+\frac{1}{n^{2}}+\cdots+\frac{1}{n^{M}}\right) \leq 2\left(P_{1}-P_{0}\right) / n$ from one of its preimages in $\phi_{e_{k}}^{n}(A)$.

The following lemma is the main step in the proof of Proposition 1.1.

Lemma 1.4. Let $R_{0}, R_{1}$ be numbers such that $1 / 2 \leq R_{0}<R_{1} \leq 2 R_{0} \leq 2$, $n$ a positive integer, and let $M \approx n^{\frac{d-1}{2}}$ be the cardinality of $D$, the maximal $\frac{1}{C \sqrt{n}}$-separated subset of $S^{d-1}$. Then any annulus $\left\{x \in \mathbb{R}^{d}: R_{0} \leq\left|x-x_{0}\right| \leq\right.$ $\left.R_{1}\right\}$ may be divided into $n^{M}$ closed subannuli, each of width $\left(R_{1}-R_{0}\right) n^{-M}$, which when properly translated about yield a compact set $E_{n}$ such that $\left|E_{n}\right| \lesssim$ $\left(R_{1}-R_{0}\right) / n$. Moreover $E_{n} \subset\left\{x \in \mathbb{R}^{d}: R_{0}-\left(R_{1}-R_{0}\right) \leq\left|x-x_{0}\right| \leq R_{1}\right\}$.

Proof. Without loss of generality we may assume that $x_{0}=0$. Define $A_{0}=\left\{x \in \mathbb{R}^{d}: R_{0} \leq|x| \leq R_{1}\right\}$, and for $k=1,2, \ldots, M$ let $A_{k}=$ $\phi_{e_{k}}^{n}\left(A_{k-1}\right)$; take $E_{n}=A_{M}$. Clearly $E_{n}$ is a compact subset which is the union of $n^{M}$ annuli of width $\left(R_{1}-R_{0}\right) n^{-M}$. Also, since in general for an annulus $A=\left\{x \in \mathbb{R}^{d}: P_{0} \leq\left|x-x_{0}\right| \leq P_{1}\right\}, \quad \phi_{e}^{n}(A) \subset\left\{x \in \mathbb{R}^{d}\right.$ : $\left.P_{0}-\frac{n-1}{n}\left(P_{1}-P_{0}\right) \leq\left|x-x_{0}\right| \leq P_{1}\right\}$, we see that $E_{n} \subset\left\{x \in \mathbb{R}^{d}\right.$ : $\left.R_{0}-\left(R_{1}-R_{0}\right) \leq\left|x-x_{0}\right| \leq R_{1}\right\}$.

To obtain the desired upper bound for $\left|E_{n}\right|$ we devise a cover of $E_{n}$ by sets whose measure we may readily estimate.

To facilitate the construction of this cover we must delineate the $n^{k-1}$ figures of $A_{k}$. To each annulus of $E_{n}$ we associate uniquely a multi-index $I$, the address of the annulus, as follows. For a given annulus of $E_{n}$ its center can be written

$$
\left(P_{1}-P_{0}\right) \sum_{j=1}^{M} i_{j} \frac{e_{j}}{n^{j}} \quad e_{j} \in D,
$$

where the coefficients $i_{j}$ are integers in $[0, n-1]$ and hence uniquely given; the address for this annulus is then $I=\left(i_{1}, i_{2}, \ldots, i_{M}\right)$. Denote the center of this annulus as $x(I)$. Let $S_{k}$ denote the set of indices of the form $\left(i_{1}, \ldots, i_{k-1}, 0, \ldots, 0\right){ }^{2}$ It is easy to see that $x(I), I \in S_{k}$, is the center of a figure of $A_{k}$; denote this figure as $A_{k}(x(I))$. With this notation we remark that if $z \in E_{n}$ is contained in an annulus with address $\left(i_{1}, i_{2}, \ldots, i_{M}\right)$, then $z \in A_{M}\left(x\left(i_{1}, \ldots, i_{M-1}, 0\right)\right)$, and for any $k=1, \ldots M$ and any $I \in S_{k}$, $z \in \phi_{n}^{e_{M}} \circ \cdots \circ \phi_{n}^{e_{k+1}}\left(A_{k}(x(I))\right)$.

\footnotetext{
${ }^{2}$ Here $S_{1}=\{(0, \ldots, 0)\}$
} 
For each $k=1,2, \ldots, M$ and for each $I \in S_{k}$ we take as an element of our proposed cover of $E_{n}$

$$
N_{2\left(R_{1}-R_{0}\right) / n^{k}}\left(A_{k}(x(I)) \cap \Gamma_{2 / \sqrt{n}}^{e_{k}}(x(I))\right) .
$$

By Lemma 1.2 with $P_{1}-P_{0}=\left(R_{1}-R_{0}\right) / n^{k-1}$,

$$
\left|N_{2\left(R_{1}-R_{0}\right) / n^{k}}\left(A_{k}(x(I)) \cap \Gamma_{2 / \sqrt{n}}^{e_{k}}(x(I))\right)\right| \lesssim \frac{1}{n^{k-1}} \cdot \frac{\left(R_{1}-R_{0}\right)}{n^{\frac{d+1}{2}}},
$$

and so

$$
\left|\bigcup_{I \in S_{k}} N_{2\left(R_{1}-R_{0}\right) / n^{k}}\left(A_{k}(x(I)) \cap \Gamma_{2 / \sqrt{n}}^{e_{k}}(x(I))\right)\right| \lesssim \frac{\left(R_{1}-R_{0}\right)}{n^{\frac{d+1}{2}}} .
$$

The claim is that the collection of all sets defined in (8) covers $E_{n}$. Let $z \in E_{n}$ be given and choose an index $I=\left(i_{1}, \ldots, i_{M-1}, 0\right) \in S_{M}$ such that $z \in A_{M}(x(I))$-i.e., find one of the $n^{M-1}$ figures of $E_{n}$ that $z$ lies in. If $C$ is large enough then

$$
\begin{aligned}
\mathbb{R}^{d}= & \Gamma_{1 / \sqrt{n}}^{e_{1}}(0) \cup \Gamma_{1 / \sqrt{n}}^{e_{2}}\left(x\left(i_{1}, 0 \ldots, 0\right)\right) \cup \Gamma_{1 / \sqrt{n}}^{e_{3}}\left(x\left(i_{1}, i_{2}, 0 \ldots, 0\right)\right) \cup \cdots \\
& \cup \Gamma_{1 / \sqrt{n}}^{e_{M}}\left(x\left(i_{1}, \ldots, i_{M-1}, 0\right)\right) .
\end{aligned}
$$

This is easy to see if one remembers that $x\left(i_{1}, \ldots, i_{k}, 0, \ldots 0\right)$ and $x\left(i_{1}, \ldots\right.$, $\left.i_{k+1}, 0, \ldots, 0\right)$ are very close together when $k \geq 1$.

So choose $k$ such that $z \in \Gamma_{2 / \sqrt{n}}^{e_{k}}\left(x\left(i_{1}, \ldots, i_{k-1}, 0, \ldots, 0\right)\right)$. The claim is that

$$
z \in N_{\frac{2\left(R_{1}-R_{0}\right)}{n^{k}}}\left(A_{k}\left(x\left(i_{1}, \ldots, i_{k-1}, 0, \ldots, 0\right)\right) \cap \Gamma_{2 / \sqrt{n}}^{e_{k}}\left(x\left(i_{1}, \ldots, i_{k-1}, 0, \ldots, 0\right)\right)\right) .
$$

This is established by Lemma 1.3 with $P_{1}-P_{0}=\left(R_{1}-R_{0}\right) / n^{k-1}$ once we recall, given the above remarks, that for this choice of $k, z \in \phi_{n}^{e_{M}} \circ \cdots \circ$ $\phi_{n}^{e_{k+1}}\left(A_{k}(x i k)\right)$.

Finally by $(9)$

$$
\begin{aligned}
\left|E_{n}\right| & \leq \sum_{k=1}^{M} \sum_{I \in S_{k}}\left|N_{2\left(R_{1}-R_{0}\right) / n^{k}}\left(A_{k}(x(I)) \cap \Gamma_{2 / \sqrt{n}}^{e_{k}}(x(I))\right)\right| \\
& \lesssim\left(R_{1}-R_{0}\right)(\underbrace{n^{-\frac{d+1}{2}}+n^{-\frac{d+1}{2}}+\cdots+n^{-\frac{d+1}{2}}}_{M \text { times }}) \approx\left(R_{1}-R_{0}\right) n^{-1},
\end{aligned}
$$

which establishes the lemma.

The rest of the proof of Proposition 1.1 follows a standard pattern, and furthermore is identical to the corresponding argument in [1], so we will omit it. 


\section{The higher dimensional case.}

The purpose of this section is to prove Theorem $1^{\prime}$. Before doing so we show that the result is sharp except for the logarithmic factor (for this factor, see Proposition 2.2).

Proposition 2.1. $\left\|M_{\delta}\right\|_{L^{p} \rightarrow L^{q}} \gtrsim \max \left\{\delta^{-\left(\frac{1}{p}-\frac{1}{p^{\prime}}\right)}, \delta^{-\left(\frac{1}{p}-\frac{1}{q}\right)}\right\}$.

Proof. Let $E_{\delta}$ be a $C^{-1} \delta$-neighborhood of the set $\left\{x \in \mathbb{R}^{d}: x_{d}=0,|x|=\right.$ $1 / 2\}$, and $f=\chi_{E_{\delta}}$. Then $\|f\|_{p}=\left|E_{\delta}\right|^{1 / p} \approx \delta^{2 / p}$. If $C$ is large then $E_{\delta}$ is contained in $C_{\delta}\left(y_{r}, r\right)$ for any $r \in[1 / 2,2]$, where $y_{r}=\left(0, \ldots, 0, \sqrt{r^{2}-1 / 4}\right) \in$ $\mathbb{R}^{d}$, and therefore $M_{\delta} f(r) \geq \frac{\left|E_{\delta}\right|}{\left|C_{\delta}\left(y_{r}, r\right)\right|} \approx \delta=\delta^{-\left(\frac{1}{p}-\frac{1}{p^{\prime}}\right)}\|f\|_{p}$ for every $r$. On the other hand let $f=\chi_{C_{\delta}(0,1)}$. Then $\|f\|_{p}=\delta^{1 / p}$, while for $r \in$ $[1-\delta, 1+\delta] M_{\delta} f(r) \approx 1$. Thus $\left\|M_{\delta} f\right\|_{q} \gtrsim \delta^{1 / q}=\delta^{1 / q-1 / p}\|f\|_{p}$.

The proof of Theorem 1' necessitates a lemma, whose proof we omit.

Lemma 2.1. If $d \geq 3,1 / 2 \leq r, s \leq 2$, then for any $a, b \in \mathbb{R}^{d}$ we have

$$
\left|C_{\delta}(a, r) \cap C_{\delta}(b, s)\right| \leq C \frac{\delta^{2}}{|r-s|+\delta} .
$$

Proof of Theorem $1^{\prime}$. The $L^{p} \rightarrow L^{\infty}$ case is trivial. By interpolation and by Hölder's inequality it suffices to prove the theorem in the $L^{p} \rightarrow L^{p^{\prime}}$ case, $1<p \leq 2$.

First we "discretize": If $|s-r|<\delta$ then $M_{\delta} f(s) \lesssim M_{3 \delta} f(r)$ since $C_{\delta}(x, s) \subset$ $C_{3 \delta}(x, r)$. Consequently it suffices to show that

$$
\begin{aligned}
& \left(\sum_{j} \delta\left|M_{\delta} f\left(r_{j}\right)\right|^{p^{\prime}}\right)^{\frac{1}{p^{\prime}}} \lesssim \delta^{1-\frac{2}{p}}\|f\|_{p}, \quad 1<p<2 \\
& \left(\sum_{j} \delta\left|M_{\delta} f\left(r_{j}\right)\right|^{2}\right)^{\frac{1}{2}} \lesssim\left(\log \left(\frac{1}{\delta}\right)\right)^{\frac{1}{2}}\|f\|_{2},
\end{aligned}
$$

where say $r_{j}=1 / 2+j \delta, 0 \leq j \leq 3 / 2 \delta$.

Next we use duality: For suitable nonnegative numbers $a_{j}$ with $\sum \delta a_{j}^{p}=1$ and suitable points $x_{j} \in \mathbb{R}^{d}$ we have (with $1<p \leq 2$ )

$$
\begin{aligned}
\left(\sum_{j} \delta\left|M_{\delta} f\left(r_{j}\right)\right|^{p^{\prime}}\right)^{1 / p^{\prime}} & =\sum_{j} \delta a_{j} M_{\delta} f\left(r_{j}\right) \\
& =\int f \cdot \sum_{j} b_{j} \chi_{j}
\end{aligned}
$$


where $b_{j}=\frac{\delta a_{j}}{\left|C_{\delta}\left(x_{j}, r_{j}\right)\right|}$ satisfies $\sum \delta b_{j}^{p} \sim \sum \delta a_{j}^{p}=1$, and $\chi_{j}=\chi_{C_{\delta}\left(x_{j}, r_{j}\right)}$. It therefore suffices by Hölder's inequality to show that

$$
\begin{aligned}
& \left\|\sum_{j} b_{j} \chi_{j}\right\|_{p^{\prime}} \lesssim \delta^{1-2 / p}\left(\sum_{j} \delta b_{j}^{p}\right)^{1 / p}, \quad 1<p<2 \\
& \left\|\sum_{j} b_{j} \chi_{j}\right\|_{2} \lesssim(\log (1 / \delta))^{1 / 2}\left(\sum_{j} \delta b_{j}^{2}\right)^{1 / 2} .
\end{aligned}
$$

Let $\chi_{j k}=\chi_{C_{\delta}\left(x_{j}, r_{j}\right) \cap C_{\delta}\left(x_{k}, r_{k}\right)}$. For $1<p \leq 2$

$$
\begin{aligned}
\left\|\sum_{j} b_{j} \chi_{j}\right\|_{p^{\prime}}^{2} & \left.=\iint\left|\sum_{j k} b_{k} b_{j} \chi_{j k}(y)\right|^{p^{\prime} / 2} d y\right)^{2 / p^{\prime}} \\
& \leq \sum_{j k} b_{k} b_{j}\left(\int\left|\chi_{j k}(y)\right|^{p^{\prime} / 2} d y\right)^{2 / p^{\prime}} \\
& \lesssim \sum_{j k} b_{k} b_{j}\left(\frac{\delta}{1+|j-k|}\right)^{2 / p^{\prime}} \quad \text { by Lemma } 2.1 \\
& \leq \delta^{2(1-2 / p)}\left(\sum_{k} \delta b_{k}^{p}\right)^{1 / p}\left\|\sum_{j} \delta^{1 / p} b_{j}(1+|j-k|)^{-2 / p^{\prime}}\right\|_{l^{\prime}} .
\end{aligned}
$$

On the one hand, by the Hardy-Littlewood-Sobolev inequality for the integers $^{3}$, when $1<p<2$,

$$
\left\|\sum_{j} \delta^{1 / p} b_{j}(1+|j-k|)^{-2 / p^{\prime}}\right\|_{l^{p^{\prime}}} \lesssim\left(\sum_{j} \delta b_{j}^{p}\right)^{1 / p} .
$$

One the other hand, by Schur's test

$$
\left\|\sum_{j} \delta^{1 / 2} b_{j}(1+|j-k|)^{-1}\right\|_{l^{2}} \lesssim(\log (1 / \delta))^{1 / 2}\left(\sum_{j} \delta\left|b_{j}\right|^{2}\right)^{1 / 2},
$$

and the theorem is proved.

Proposition 2.2. A necessary condition for an estimate of the form

$$
\left\|M_{\delta} f\right\|_{L^{q}([1 / 2,2])} \leq C(\log (1 / \delta))^{\alpha}\|f\|_{L^{p}\left(\mathbb{R}^{d}\right)}
$$

to hold is that $\alpha \geq \frac{2}{(d-1) p}$.

\footnotetext{
${ }^{3}$ The referee points out that this was the original version proved by Hardy and Littlewood.
} 
Proof. Let $E_{n}$ be the set described in Lemma 1.4 (with $R_{0}=1 / 2$ and $R_{1}=2$ ). Take $\delta=n^{-M}\left(M \approx n^{\frac{d-1}{2}}\right)$ and let $f=\chi_{E_{n}}$. Then $\left|E_{n}\right| \lesssim \frac{1}{n}$, but $M_{\delta} f(r) \gtrsim$ 1 for $r \in[1 / 2,2]$. Thus

$$
1 \lesssim\left(\log \left(n^{M}\right)\right)^{\alpha} n^{-\frac{1}{p}}=n^{\frac{\alpha(d-1)}{2}-\frac{1}{p}}(\log (n))^{\alpha},
$$

which is only possible when $\alpha \geq \frac{2}{(d-1) p}$.

Remark. When $d=3$, this shows that the powers of $\log \frac{1}{\delta}$ in Theorem $1^{\prime}$ are the optimal ones. Taking $d=2, p=3$ it also gives a lower bound $\frac{2}{3}$ for the number of logarithms which would be needed in (3). Note that this shows that Theorem $1^{\prime}$ cannot hold as stated when $d=2$.

\section{Further remarks.}

(1) Theorem $1^{\prime}$ (and $2^{\prime}$ ) have equivalent formulations in terms of the maximal function $u^{*}(t)=\sup _{x}|u(x, t)|$, where $u$ is a solution of the wave equation. The argument relating the two maximal functions is a standard one and we will only sketch it. Namely, let $C_{f}(x, t)$ be the average of $f$ over the sphere centered at $x$ with radius $t$. If (in $d$ space dimensions) $u_{t t}-\Delta_{x} u=0$ and $u(\cdot, 0)=f, u_{t}(\cdot, 0)=g$, and if $\hat{f}(\xi)$ and $\hat{g}(\xi)$ vanish when $|\xi| \leq 1$ then one can obtain $u(\cdot, t)$ in the form

$$
\begin{aligned}
\mathcal{A}_{t}\left(C_{(-\Delta)} \frac{d-1}{4} f\right. & (\cdot, t))+\mathcal{B}_{t}\left(C_{(-\Delta)^{\frac{d-3}{4}} g}(\cdot, t)\right) \\
& +\mathcal{C}_{t}\left(\frac{d}{d t} C_{(-\Delta)^{\frac{d-3}{4}} f}(\cdot, t)\right)+\mathcal{D}_{t}\left(\frac{d}{d t} C_{(-\Delta) \frac{d-5}{4} g}(\cdot, t)\right)
\end{aligned}
$$

where $\mathcal{A}_{t}, \mathcal{B}_{t}, \mathcal{C}_{t}, \mathcal{D}_{t}$ are operators which are bounded on $L^{\infty}$, uniformly in $t \in\left[\frac{1}{2}, 2\right]$. This may be seen by taking Fourier transforms and using the asymptotics for the Fourier transform of surface measure and the fact that a multiplier $m$ supported outside the unit disc with $D^{\alpha} m(\xi)=\mathcal{O}\left(|\xi|^{-(|\alpha|+1)}\right)$ at $\infty$ will be $L^{\infty}$ bounded. Furthermore, by differentiation under the integral sign

$$
\left|\frac{d}{d t} C_{(-\Delta)^{\frac{d-3}{4}} f}(x, t)\right| \lesssim \sum_{i=1}^{d} C_{\left|R_{i}(-\Delta)^{\frac{d-1}{4}} f\right|}(x, t)
$$

where the $R_{i}$ are the Riesz transforms, and similarly with the fourth term in (10). It follows (with $R_{0} \stackrel{\text { def }}{=}$ identity operator) that

$$
u^{*}(t) \lesssim \sup _{x} \sum_{i=0}^{d} C_{\left|R_{i}(-\Delta)^{\frac{d-1}{4}} f\right|}(x, t)+C_{\left|R_{i}(-\Delta)^{\frac{d-3}{4}} g\right|}(x, t),
$$

so that any estimate for $M_{\delta}$ gives a corresponding estimate for $u^{*}$. For example the first statement in Theorem $1^{\prime}$ corresponds in this way to the 
estimate

$$
\left\|u^{*}\right\|_{L^{2}\left(\left[\frac{1}{2}, 2\right]\right)} \lesssim\|f\|_{\alpha}+\|g\|_{\alpha-1}, \quad \alpha>\frac{d-1}{2}, \quad d \geq 3 .
$$

Here \|\|$_{\alpha}$ is the inhomogeneous $L^{2}$ Sobolev space norm with $\alpha$ derivatives. One would expect (11) to be a well-known estimate, although we only know a reference in the three dimensional case (namely [9]).

(2) One can also extend the domain of $M_{\delta} f$ from $[1 / 2,2]$ to $(0, \infty)$. This of course requires modifying the definition of $M_{\delta}$. We let

$$
C_{\delta}^{\prime}(x, r)=\left\{z \in \mathbb{R}^{d}: r(1-\delta) \leq|z-x| \leq r(1+\delta)\right\} .
$$

Then if $f: \mathbb{R}^{d} \rightarrow \mathbb{R}$ we define $M_{\delta}{ }^{\prime} f:(0, \infty) \rightarrow \mathbb{R}$ via

$$
M_{\delta}^{\prime} f(r)=\sup _{x} \frac{1}{\left|C_{\delta}^{\prime}(x, r)\right|} \int_{C_{\delta}^{\prime}(x, r)}|f| .
$$

Theorem $\mathbf{1}^{\prime \prime}$. Assume $d \geq 3$. Then

$$
\left(\int_{0}^{\infty}\left|M_{\delta}^{\prime} f(r)\right|^{2} r^{d} \frac{d r}{r}\right)^{1 / 2} \leq C \log \left(\frac{1}{\delta}\right)^{\frac{3}{2}}\|f\|_{L^{2}\left(\mathbb{R}^{d}\right)} .
$$

If $1<p<2$ then

$$
\left(\int_{0}^{\infty}\left|M_{\delta}^{\prime} f(r)\right|^{p^{\prime}} r^{\frac{d}{p-1}} \frac{d r}{r}\right)^{1 / p^{\prime}} \leq C_{p} \delta^{-\left(\frac{1}{p}-\frac{1}{p^{\prime}}\right)}\|f\|_{L^{p}\left(\mathbb{R}^{d}\right)} .
$$

This is proved in a standard way using Theorem $1^{\prime}$ and a small amount of Littlewood-Paley theory.

\section{Proof of Theorem $2^{\prime}$, part 1.}

Before giving the proof of Theorem $2^{\prime}$ we want to make some remarks concerning the difference between the two- and higher-dimensional cases. Note to begin with that Lemma 2.1 fails in the two dimensional case: The corresponding estimate is

$$
\left|C_{\delta}(x, r) \cap C_{\delta}(y, s)\right| \lesssim \frac{\delta^{\frac{3}{2}}}{(|r-s|+\delta)^{\frac{1}{2}}} .
$$

This is sharp when the circles $\{z:|z-x|=r\}$ and $\{z:|z-y|=s\}$ are tangent. The bound (12) leads via the argument of Section 2 to the estimate

$$
\left\|M_{\delta} f\right\|_{2} \lesssim \delta^{-\frac{1}{4}}\|f\|_{2} .
$$

This is the optimal $L^{2} \rightarrow L^{2}$ estimate (by the example in the next paragraph) but implies only the bound $\operatorname{dim} E \geq \frac{3}{2}$ for the Hausdorff dimension of a set containing a circle of every radius. 
To see why (3) is the natural conjecture to make, let $E_{\delta}$ be a $\delta \times \delta^{\frac{1}{2}}$ rectangle and let $f$ be the characteristic function of $E_{\delta}$. For any $r \in\left[\frac{1}{2}, 2\right]$ there is an annulus $C_{\delta}(x, r)$ which contains a fixed fraction of $E_{\delta}$ (namely: Choose $x$ so that the circle $\{z:|z-x|=r\}$ is tangent to a long side of $E_{\delta}$ ) and therefore $M_{\delta} f(r) \gtrsim \delta^{\frac{1}{2}}$. It follows that a bound

$$
\forall \epsilon \exists C_{\epsilon}:\left\|M_{\delta} f\right\|_{p} \leq C_{\epsilon} \delta^{-\epsilon}\|f\|_{p}
$$

can only hold if $\delta^{\frac{1}{2}} \leq \delta^{\frac{3}{2 p}}$, i.e., $p \geq 3$. Similar considerations show that the bound (4) would be best possible for each $p$ and $q$, and the example $f=$ characteristic function of $C_{\delta}(0,1)$ shows that the range $q \leq 2 p^{\prime}$ in (4) would also be best possible.

As we pointed out in the introduction, the $p=2, q=4$ case of Theorem $2^{\prime}$ is due to Pecher [9]. When $p>2$ it is natural to use a geometric argument in order to deal with the problems arising from tangent circles. Our way of doing this will be based on a geometric fact used also by Marstrand [7], together with a well-known argument from elementary combinatorics. In order to avoid certain irrelevant technicalities, let us say that two circles are internally tangent if they are tangent and one is contained in the bounded component of the complement of the other. Then we have the following geometrical fact, which we call (M), and which we believe was first used in a similar context in [7]: Given three circles $C_{1}, C_{2}, C_{3}$, there are at most two circles which are internally tangent to $C_{1}, C_{2}$ and $C_{3}$ at three different points. It is easy to see that this leads to control over the number of possible tangencies among $N$ circles, $N$ large:

Proposition 3.1. Let $C_{1}, \ldots, C_{N}$ are circles. Let $k$ be a number and assume: For each of at least $\frac{N}{2}$ values of $j$, we are given $k$ values of $i$ such that $C_{i}$ is internally tangent to $C_{j}$ with (for fixed $j$ ) no more than $\frac{k}{3}$ of these tangencies occurring at any one point. Then $k \lesssim N^{\frac{2}{3}}$.

Proof. This basically follows by combining fact (M) with [4], Theorem 3 on p. 111: An $N \times N(0,1)$ matrix with no $3 \times 3$ submatrix of 1's contains $\lesssim N^{\frac{5}{3}} 1$ 's altogether. However, we have stated the proposition in a way which makes the result from [4] not directly applicable, so we give a proof (assuming (M)). Let $Q$ be the set of all quadruples $\left(j, i_{1}, i_{2}, i_{3}\right)$ such that $C_{j}$ is internally tangent to $C_{i_{1}}, C_{i_{2}}$ and $C_{i_{3}}$ at three different points. Then $\operatorname{card}(Q) \leq 2 N^{3}$, since for any given $i_{1}, i_{2}, i_{3},(\mathrm{M})$ implies there are at most two choices for $j$. On the other hand, $\operatorname{card}(Q) \geq \frac{N}{2} \cdot k \cdot \frac{2 k}{3} \cdot \frac{k}{3}$, since if we take $j$ to be one of the $\frac{N}{2}$ values in the statement, then there are at least $k$ possibilities for $i_{1}$, and then $k-\frac{k}{3}$ possibilities for $i_{2}$ and $k-\frac{2 k}{3}$ possibilities for $i_{3}$. Hence $2 N^{3} \geq \frac{N k^{3}}{9}$ and the proposition follows. 
The proof of Theorem $2^{\prime}$ will be basically a quantitative version of the proof of Proposition 3.1. We note that the example of circles with integer radius $\left(\leq n^{\frac{1}{3}}\right)$ and center at an integer lattice point (lying in $D\left(0, n^{\frac{1}{3}}\right)$ ) shows that the optimal exponent to replace $\frac{2}{3}$ in the proposition must be at least $\frac{1}{3}$, since tangencies may be identified with pythagorean triples.

Added 5/15/97. More sophisticated methods for dealing with combinatorial problems like the one in Proposition 3.1 have been developed in the discrete geometry literature and can be applied in connection with (3). See the remark at the end of the introduction.

In fact, as mentioned in the introduction we will work in the general situation of a family of curves satisfying the "cinematic curvature hypothesis" from [10], instead of with circles.

Let $\Phi: U \rightarrow \mathbb{R}, U$ a neighborhood of $(a, b)$ in $\mathbb{R}^{2} \times \mathbb{R}^{2}$ be a function satisfying the following conditions:

$$
\nabla_{x} \Phi_{\left.\right|_{(x, y)=(a, b)}} \neq 0 .
$$

If $e$ is a unit vector with $e \perp \nabla_{x} \Phi(a, b)$,

$$
\text { then } d_{y}\left(\begin{array}{c}
\nabla_{x} \Phi \cdot e \\
\frac{\nabla_{x}^{2} \Phi(e, e)}{\left|\nabla_{x} \Phi\right|}
\end{array}\right)_{\left.\right|_{(x, y)=(a, b)}} \text { is invertible. }
$$

We use the notation $d_{y} f$ for the differential of $f$ with respect to $y$ and also use $\nabla_{y} \Phi$ interchangeably with $d_{y} \Phi$ if $\Phi$ maps into $\mathbb{R}$, and $\nabla_{y}^{2} \Phi$ for the Hessian of $\Phi$ in the $y$ variable, etc.

We define

$$
C(y, r)=\left\{x \in \mathbb{R}^{2}: \Phi(x, y)=r\right\} .
$$

Condition (15) has a number of equivalent formulations, cf. [10], [11]. We recall: If $y$ and $z$ are close to $b$ and $C(y, r)$ and $C(z, s)$ intersect near $a$ then (since $\Phi$ is smooth) $|r-s| \lesssim|y-z|$. If we assume (14) then (locally) the $C(y, r)$ are smooth curves, and (15) is equivalent to the following condition: If $|y-b|,|r-\Phi(a, b)|,|z-b|,|s-\Phi(a, b)|$ and $|x-a|$ are small and $C(y, r)$ and $C(z, s)$ intersect at $x$ then either the unit tangent vectors to $C(y, r)$ and $C(z, s)$ at $x$, or else their curvatures at $x$, must differ by $\gtrsim|y-z|+|r-s|$.

In what follows, $\mathcal{Q}(\beta)$ will always mean the set $\left\{(z, s, y, r) \in \mathbb{R}^{2} \times \mathbb{R} \times \mathbb{R}^{2} \times\right.$ $\mathbb{R}: \max (|z-b|,|s-\Phi(a, b)|,|y-b|,|r-\Phi(a, b)|)<\beta\}$. We fix small positive numbers $\alpha_{2} \ll \alpha_{1} \ll \alpha_{0}$ and $\beta_{0}$, and define a function $\Delta: \mathcal{Q}\left(\beta_{0}\right) \rightarrow \mathbb{R}$ via

$$
\Delta(z, s, y, r)=\inf _{\substack{x \in C(y, r) \cap D\left(a, \alpha_{1}\right) \\ x^{\prime} \in C(z, s) \cap D\left(a, \alpha_{1}\right)}}\left|x-x^{\prime}\right|+\left|\frac{\nabla_{x} \Phi(x, y)}{\mid \nabla_{x} \Phi(x, y)}-\frac{\nabla_{x} \Phi\left(x^{\prime}, z\right)}{\left|\nabla_{x} \Phi\left(x^{\prime}, z\right)\right|}\right| .
$$


Thus $\Delta(z, s, y, r)$ measures the minimum distance between the curves obtained by lifting $C(z, s)$ and $C(y, r)$ to the unit tangent bundle. We further define (for small $\delta$ )

$$
C_{\delta}(y, r)=\left\{x \in D\left(0, \alpha_{2}\right):|\Phi(x, y)-r|<\delta\right\} .
$$

The constants in Lemma 3.1 below are of course independent of $\delta, \epsilon, t$ and $\lambda$.

Lemma 3.1 below is the main result of this section. In $(19), N_{\delta}\left(S^{*}\right)$ is the $\delta$-entropy of $S^{*}$, i.e., the maximum possible cardinality for a $\delta$-separated subset of $S$. Part (i) and estimate (19) are what are needed in the subsequent sections; the remaining statements follow from the same proof and are included for the sake of possible future applications.

Lemma 3.1. There are $\beta_{1}>0$ and $A_{1}<\infty$ such that if $\delta>0$ is small enough then:

(i) If $(z, s, y, r) \in \mathcal{Q}\left(\beta_{1}\right)$ then

$$
\begin{gathered}
\left|C_{\delta}(z, s) \cap C_{\delta}(y, r)\right| \lesssim \frac{\delta^{2}}{\sqrt{(|y-z|+|r-s|+\delta)(\Delta(z, s, y, r)+\delta)}} \\
\quad \operatorname{diam}\left(C_{\delta}(z, s) \cap C_{\delta}(y, r)\right) \lesssim \sqrt{\frac{\Delta(z, s, y, r)+\delta}{|y-z|+|r-s|+\delta}} .
\end{gathered}
$$

(ii) Let $\epsilon, t, \lambda \in(0,1)$ be such that $\epsilon \geq \delta$ and $\lambda \sqrt{\frac{t}{\epsilon}} \geq A_{1}$. For fixed $\left(z_{i}, r_{i}\right) \epsilon$ $\mathbb{R}^{2} \times \mathbb{R}, i=1,2,3$ with $\max \left(\left|z_{i}-b\right|,\left|r_{i}-\Phi(a, b)\right|\right)<\beta_{1}$, define

$$
\begin{aligned}
& S=\left\{(y, r) \in \mathbb{R}^{2} \times \mathbb{R}:\left(z_{i}, r_{i}, y, r\right) \in \mathcal{Q}\left(\beta_{1}\right), \Delta\left(z_{i}, r_{i}, y, r\right)<\epsilon \forall i,\right. \\
& \quad\left|y-z_{i}\right|+\left|r-r_{i}\right|>t \forall i, C_{\delta}\left(z_{i}, r_{i}\right) \cap C_{\delta}(y, r) \neq \emptyset \forall i, \\
& \left.\quad \operatorname{dist}\left(C_{\delta}\left(z_{i}, r_{i}\right) \cap C_{\delta}(y, r), C_{\delta}\left(z_{j}, r_{j}\right) \cap C_{\delta}(y, r)\right)>\lambda \forall(i, j): i \neq j\right\} .
\end{aligned}
$$

Then $S$ is the union of two sets, each of volume $\lesssim \frac{\epsilon^{3}}{\lambda^{3}}$ and diameter $\lesssim \frac{\epsilon}{\lambda^{2}}$. In particular, if $S^{*}=\left\{r \in \mathbb{R}:(y, r) \in S\right.$ for some $\left.y \in \mathbb{R}^{2}\right\}$, then

$$
N_{\delta}\left(S^{*}\right) \lesssim \frac{\epsilon}{\delta \lambda^{2}}
$$

\section{Remarks.}

(1) The main point is part (ii); part (i) is implicit in [10] (as are several of the sublemmas below) and in any case is quite simple.

(2) The case of circles corresponds of course to $\Phi(x, y)=|x-y|, a \neq b$. The function $\Delta(z, s, y, r)$ then satisfies $\Delta(z, s, y, r) \gtrsim \| z-y|-| r-s||$. In this case Lemma 3.1 is essentially in [7] (cf. Lemma 5.2 there), but some of the conclusions of Lemma 3.1 are not stated there. It may therefore be worth recording the following "explicit" form of Lemma 3.1 (ii) for circles even though we will not use it below. It is not quite a formal consequence 
of Lemma 3.1 but can be proved by a much abbreviated version of the proof of Lemma 3.1 - as has already mentioned, it is also implicit in [7].

Fix three circles $C\left(x_{i}, r_{i}\right) \stackrel{\text { def }}{=}\left\{z \in \mathbb{R}^{2}:\left|z-x_{i}\right|=r_{i}\right\}$, where $r_{i} \in\left[\frac{1}{2}, 2\right]$, and let $C_{\delta}(x, r)=\left\{z \in \mathbb{R}^{2}: r-\delta<|z-x|<r+\delta\right\}$. Fix $\epsilon, t, \lambda \in$ $(0,1)$ such that $\epsilon \geq \delta$ and $\lambda \sqrt{\frac{t}{\epsilon}} \geq A_{1}$. Define two annuli $C_{\delta}(x, r)$ and $C_{\delta}(y, s)$ to be interior $(\epsilon, t)$-tangent if their intersection is nonempty and also ||$x-y|-\overline{|r-s| \mid<\epsilon \text { and }|x-y|}+| r-s \mid>t$. Let $\Omega_{\epsilon t \lambda}$ be the set of all $(x, r) \in \mathbb{R}^{2} \times\left[\frac{1}{2}, 2\right]$ such that $C_{\delta}(x, r)$ is interior $(\epsilon, t)$-tangent to all three annuli $C_{\delta}\left(x_{i}, r_{i}\right)$ and futhermore the distance between any two of the sets $C_{\delta}\left(x_{i}, r_{i}\right) \cap C_{\delta}(x, r)$ and $C_{\delta}\left(x_{j}, r_{j}\right) \cap C_{\delta}(x, r)(1 \leq i, j \leq 3, i \neq j)$ is at least $\lambda$. Then $\Omega_{\epsilon t \lambda}$ is contained in the union of two ellipsoids in $\mathbb{R}^{3}$ each of volume $\lesssim \frac{\epsilon^{3}}{\lambda^{3}}$ and diameter $\lesssim \frac{\epsilon}{\lambda^{2}}$.

(3) In the proof of Lemma 3.1 we often assume that $a=b=0$ and $\Phi(a, b)=0$.

We start the proof by making some further observations about the conditions (14), (15). First, it is clear that

$$
C_{\delta}(z, s) \cap C_{\delta}(y, r)=\emptyset \text { if }|r-s|>C|y-z|+2 \delta
$$

for a suitable constant $C$. (14), (15) are invariant under changes of coordinates in the $x$ variable, so for many purposes we may assume that $\Phi(x, 0)=x_{(2)}$. Here we use the notation $x_{(2)}$ to mean the second coordinate of the point $x \in \mathbb{R}^{2}$. If $\Phi(x, 0)=x_{(2)}$, then (15) is equivalent with

$$
C^{-1}|y| \leq\left|\frac{\partial \Phi}{\partial x_{(1)}}\right|+\left|\frac{\partial^{2} \Phi}{\partial x_{(1)}^{2}}\right| \leq C|y|
$$

for suitable $C$, locally near $(0,0) \in \mathbb{R}^{2} \times \mathbb{R}^{2}$. Next we have the following (perhaps well-known) fact, which will be used in the last part of the proof of Lemma 3.1.

Lemma 3.2. Assume that $\Phi$ satisfies (14), (15). Suppose that $x_{1}, x_{2}, x_{3}, y \in$ $\mathbb{R}^{2}$ are sufficiently close to the origin and satisfy $\Phi\left(x_{1}, y\right)=\Phi\left(x_{2}, y\right)=$ $\Phi\left(x_{3}, y\right)$. Then

$$
\left|\operatorname{det}\left(\begin{array}{ll}
-\nabla_{y} \Phi\left(x_{1}, y\right) & 1 \\
-\nabla_{y} \Phi\left(x_{2}, y\right) & 1 \\
-\nabla_{y} \Phi\left(x_{3}, y\right) & 1
\end{array}\right)\right| \approx\left|x_{1}-x_{2}\right|\left|x_{2}-x_{3}\right|\left|x_{3}-x_{1}\right| .
$$

Proof. We may assume that $y=0$ and $\Phi(x, 0)=x_{(2)}$, and furthermore that $x_{1}=(0,0), x_{2}=(\sigma, 0), x_{3}=(\tau, 0)$, with $\sigma<0<\tau$. Define $v(t)=$ $\nabla_{y} \Phi((t, 0), 0)$. Thus $v(t)$ is an $\mathbb{R}^{2}$-valued function on an interval. Condition (15) implies that

$$
\dot{v}(0) \wedge \ddot{v}(0) \neq 0
$$


where we have denoted $\frac{d v}{d t}$ by $\dot{v}$ etc. Consequently, by Taylor's theorem,

$$
\begin{aligned}
& v(\tau)-v(0)=\tau \dot{v}(0)+\frac{\tau^{2}}{2} \ddot{v}(0)+\mathcal{O}\left(\tau^{3}\right) \\
& v(\sigma)-v(0)=\sigma \dot{v}(0)+\frac{\sigma^{2}}{2} \ddot{v}(0)+\mathcal{O}\left(|\sigma|^{3}\right),
\end{aligned}
$$

and therefore

$$
\begin{aligned}
\left|\operatorname{det}\left(\begin{array}{ll}
-\nabla_{y} \Phi\left(x_{1}, 0\right) & 1 \\
-\nabla_{y} \Phi\left(x_{2}, 0\right) & 1 \\
-\nabla y \Phi\left(x_{3}, 0\right) & 1
\end{array}\right)\right| & =|(v(\tau)-v(0)) \wedge(v(\sigma)-v(0))| \\
& =\frac{1}{2} \tau|\sigma|(\tau-\sigma)|\dot{v}(0) \wedge \ddot{v}(0)|+\mathcal{O}\left(|\tau \sigma|\left(\tau^{2}+\sigma^{2}\right)\right) .
\end{aligned}
$$

The lemma follows, since $\sigma<0<\tau$ so that $\tau^{2}+\sigma^{2}$ is small compared with $\tau-\sigma$.

Next we prove an elementary lemma about functions of one variable. The implicit constants depend on $C_{0}$ only.

Lemma 3.3. Let $I \subset \mathbb{R}$ be an interval, $\tilde{I}$ the double of $I, I_{0}$ the double of $\tilde{I}$, and let $\phi$ be a smooth real-valued function on $I_{0}$. Assume that

$$
C_{0}^{-1} \rho \leq|\dot{\phi}(t)|+|\ddot{\phi}(t)| \leq C_{0} \rho
$$

for all $t \in I_{0}$. Let $\Delta=\min _{t \in \tilde{I}}(|\phi(t)|+|\dot{\phi}(t)|)$. Then provided $|I|$ and $\eta_{0}$ are small enough we have:

(i) If $\Delta \leq \eta_{0} \rho$, then $\dot{\phi}(\tau)=0$ for a unique $\tau \in I_{0}$. Furthermore $|\phi(\tau)| \lesssim$ $\Delta$.

(ii) If $\tau \in I_{0}$ and $\dot{\phi}(\tau)=0$, then

$$
|\dot{\phi}(t)| \approx \rho|t-\tau|
$$

and

$$
|\phi(t)-\phi(\tau)| \approx \rho|t-\tau|^{2}
$$

for all $t \in I_{0}$.

(iii) $n(\phi, c) \leq 2$ for all $c \in \mathbb{R}$.

(iv) Let $E=\left\{(t, y) \in \mathbb{R}^{2}: t \in I:|\phi(t)|<\delta\right.$, and $\left.|y|<\delta\right\}$. Then $|E| \lesssim \frac{\delta^{2}}{\sqrt{(\rho+\delta)(\Delta+\delta)}}$ and $\operatorname{diam}(E) \lesssim \sqrt{\frac{\Delta+\delta}{\rho+\delta}}$. If $\Delta \lesssim \eta_{0} \rho$, then $E$ is contained in a disc of radius $\lesssim \sqrt{\frac{\Delta+\delta}{\rho+\delta}}$ centered at $(\tau, \phi(\tau))$. 
In (iii) $n(\phi, c)$ is the multiplicity of $c$ as a value of $\phi$, defined in the usual way, i.e.,

$$
n(\phi, c)=\sum_{t \in I_{0}: \phi(t)=c} \min \left(\left\{k \geq 1: \phi^{(k)}(t) \neq 0\right\}\right),
$$

where the minimum of the empty set is $+\infty$. We will also denote $n(\phi, 0)$ by $n(\phi)$.

Proof. (i) If $\eta_{0}+C_{0}\left|I_{0}\right| \leq \frac{1}{2} C_{0}^{-1}$ then the assumption (22) implies that $|\dot{\phi}(s)| \leq \frac{1}{2} C_{0}^{-1} \rho$ for all $s \in I_{0}$, and therefore that $|\ddot{\phi}(s)| \geq \frac{1}{2} C_{0}^{-1} \rho$ for all $s \in I_{0}$. In particular $\ddot{\phi}$ does not change sign. If $t \in I$ with $|\dot{\phi}(t)| \leq \Delta$ then (assuming as we may that $\ddot{\phi}>0$ ) we have $\frac{\dot{\phi}(s)-\dot{\phi}(t)}{s-t} \geq \frac{1}{2} C_{0}^{-1} \rho$ for all $s \in I_{0}$. If $\frac{\left|I_{0}\right|}{8 C_{0}} \geq \eta_{0}$ then it follows that $\dot{\phi}$ changes sign, i.e., we have proved the existence part of (i). We will prove the rest of (i) after proving (ii).

(ii) If $\tau$ is as in (ii) then by the same argument as above $|\dot{\phi}(t)|=\mid \dot{\phi}(t)-$ $\dot{\phi}(\tau)|\approx| t-\tau \mid \rho$, and therefore also $|\phi(t)-\phi(\tau)| \approx|t-\tau|^{2} \rho$.

The uniqueness part of (i) clearly follows from (ii). Furthermore, if $t \in$ $I$ and $|\phi(t)|+|\dot{\phi}(t)|=\Delta$, then by (ii), $|\tau-t| \lesssim \frac{\Delta}{\rho}$ and therefore also $|\phi(\tau)-\phi(t)| \lesssim \frac{\Delta^{2}}{\rho}$, so $\phi(\tau) \leq \Delta+C \frac{\Delta^{2}}{\rho} \lesssim \Delta$.

(iii) Either $\dot{\phi}$ vanishes somewhere or it doesn't. If $\dot{\phi}$ never vanishes then $n(\phi, c) \leq 1$ for all $c$. If $\dot{\phi}$ vanishes at $\tau$ then by the above considerations $\phi$ is either convex or concave and furthermore $\ddot{\phi}(\tau) \neq 0$. This implies (iii).

(iv) Let $E^{*} \stackrel{\text { def }}{=}\{t \in I:|\phi(t)|<\delta\}$, i.e., $E^{*}$ is the projection of $E$ on the $t$ axis.

We can assume that $\Delta \leq C_{0} \rho+\delta$, since otherwise $E$ is clearly empty. Given this, we may also assume that $\rho \geq 2 \eta_{0}^{-1} \delta$, since if $\Delta \lesssim \rho+\delta \lesssim \delta$ then all the statements in (iv) just follow since $E^{*} \subset I$.

Suppose first that $\Delta \leq \eta_{0} \rho$. Let $\tau$ be as in (i). If $t \in E^{*}$ then by (24),

$$
|t-\tau| \approx \sqrt{\frac{|\phi(t)-\phi(\tau)|}{\rho}} \lesssim \sqrt{\frac{\Delta+\delta}{\rho}} \approx \sqrt{\frac{\Delta+\delta}{\rho+\delta}} .
$$

This implies the last statement of (iv) and therefore also the diameter bound. If $\Delta \leq 2 \delta$ then the measure bound follows from the diameter bound and we're done. It remains to prove the measure bound when $\Delta \geq 2 \delta$. We may assume that $\tau \in \tilde{I}$, since otherwise $E$ is empty by (25) if $\eta_{0}$ has been chosen small enough. But if $\tau \in \tilde{I}$ then $|\phi(\tau)| \geq \Delta$, by definition of $\Delta$. Since $\Delta \geq 2 \delta$, it then follows by (24) that $|t-\tau| \approx \sqrt{\frac{\Delta}{\rho}}, t \in E^{*}$. Hence (by (23)) $|\dot{\phi}(t)| \approx \sqrt{\rho \Delta}$, and then the measure bound follows using (iii). 
Now suppose that $\Delta \geq \eta_{0} \rho$. Then the diameter bound in (iv) follows since $E^{*} \subset I$, and the measure bound follows (using (iii)) since $|\dot{\phi}(t)| \geq \Delta-\delta \geq$ $\eta_{0} \rho-\delta \gtrsim \rho$.

Proof of part (i) of Lemma 3.1. This follows from (iv) of Lemma 3.3 by choosing appropriate local coordinates. Namely, make a local change of variable on $D\left(0, \alpha_{0}\right)$ so that $\Phi(x, y)=x_{(2)}+r$. Then $D\left(0, \alpha_{1}\right) \cap C(y, r)$ goes over to an interval $I_{1}$ on the $x_{(1)}$ axis, $D\left(0, \alpha_{1}\right) \cap C(z, s)$ goes over to the graph of a function $x_{(2)}=\phi\left(x_{(1)}\right)$ over a (possibly slightly different) interval $I_{2}$, and the part of the graph corresponding to $D\left(0, \alpha_{2}\right)$ will lie over an interval $I$ whose double will be contained in $I_{1} \cap I_{2}$ if $\alpha_{2}$ has been chosen small enough. By (20), $\phi$ will satisfy (22) with $\rho=|y-z|$. Furthermore $\Delta(z, s, y, r)$ is clearly comparable to $\min _{s \in I_{1}}(|\phi(t)|+|\dot{\phi}(t)|+|t-s|)$, and this $t \in I_{2}$

in turn is easily seen to be comparable to $\min _{t \in I_{1} \cap I_{2}}(|\phi(t)|+|\dot{\phi}(t)|)$. So (i) of Lemma 3.1 follows by applying (iv) of Lemma 3.3 with $\tilde{I}=I_{1} \cap I_{2}$.

For future reference, we note that the above argument also shows the following: Assume that $(z, s, y, r) \in \mathcal{Q}\left(\beta_{0}\right)$ and that $\Delta(z, s, y, r)<A_{0}^{-1} \mid y-$ $z \mid$, for suitable fixed constants $\beta_{0}$ and $A_{0}$. Then there is a unique point $\xi(z, s, y) \in C(z, s) \cap D\left(0, \alpha_{0}\right)$ such that $\nabla_{x} \Phi(\xi(z, s, y), y) \wedge \nabla_{x} \Phi(\xi(z, s, y), z)=$ 0 . Furthermore we have the estimates

$$
\begin{gathered}
|\Phi(\xi(z, s, y), y)-r| \lesssim \Delta \\
C_{\delta}(y, r) \cap C_{\delta}(z, s) \subset D\left(\xi(z, s, y), C \sqrt{\frac{\Delta(z, s, y, r)+\delta}{|y-z|+\delta}}\right) .
\end{gathered}
$$

Namely, the tangency point $\xi(z, s, y)$ corresponds to the point $(\tau, \phi(\tau))$ of Lemma 3.3 under the change of variable, and the estimates follow from (i) and (iv) of Lemma 3.3 .

It remains to prove (ii) of Lemma 3.1. This will be done in two steps:

(1) There are at most two curves $C(z, s)$ which are tangent to the $C\left(z_{i}, r_{i}\right)$ at three different points.

(2) If $(y, r)$ belongs to the set $S$, then $(y, r)$ must be close to one of the points $(z, s)$ in $(1)$.

Step (1) is accomplished in the next two lemmas.

Lemma 3.4. Assume that $a_{1}, a_{2}, a_{3}, b_{1}, b_{2}, b_{3}$ are smooth functions on an interval $I_{0} \subset \mathbb{R}$, with $n\left(a_{i}-b_{j}\right) \leq 2$ for all $i$ and $j$ and $n\left(a_{i}-a_{j}\right) \leq 2$ and $n\left(b_{i}-b_{j}\right) \leq 2$ for all $i$ and $j$ with $i \neq j$. Assume furthermore that $a_{i}-b_{j}$ has a double zero for all $i$ and $j$. Then there are $i \neq j, k \neq l$, and $\tau \in I_{0}$ such that $a_{i}=a_{j}=b_{k}=b_{l}$ to second order at $\tau$. 
Remark added 5/15/97. This type of statement is well known in discrete geometry. Step (V) below is essentially the fact that a word on three letters with no abab subword has length $\leq 5$, which is a trivial case of the Davenport-Schinzel theorem.

Proof. We will argue by contradiction, so will always assume the conclusion fails.

(I) $a_{i}-b_{j}$ cannot change sign, and vanishes at just one point.

This is because $n\left(a_{i}-b_{j}\right) \leq 2$, so that the double zero of $a_{i}-b_{j}$ is its only zero.

(II) We can assume that $a_{i}-a_{j}$ and $b_{i}-b_{j}(i \neq j)$ have no double zero.

For suppose that $a_{i}=a_{j}$ to second order at $\tau \in I_{0}$. Then $a_{i}-a_{j}$ cannot change sign, and we may suppose that $a_{i}>a_{j}$ on $I_{0} \backslash\{\tau\}$. Since we are assuming the conclusion fails there is at most one $k$ such that $b_{k}(\tau)=$ $a_{i}(\tau)=a_{j}(\tau)$. Fix $l \neq k$. We can assume by symmetry that $b_{l}(\tau)>a_{i}(\tau)$. Then $b_{l} \geq a_{i}$ everywhere, hence $b_{l}>a_{j}$ everywhere, a contradiction.

(III) We can assume that there do not exist $\tau, i \neq j$ and $k$ with $a_{i}(\tau)=$ $a_{j}(\tau)=b_{k}(\tau)$ (and similarly with the roles of the $a$ 's and $b$ 's reversed).

This is because $\tau$ would have to be a double zero for $a_{i}-b_{k}$ and $a_{j}-b_{k}$ but (by (II)) a simple zero for $a_{i}-a_{j}$.

Because of (I), there is a definite ordering independent of $t \in I_{0}$ of the form

$$
\epsilon_{1} \geq \epsilon_{2} \geq \epsilon_{3} \geq \epsilon_{4} \geq \epsilon_{5} \geq \epsilon_{6}
$$

where each symbol $\epsilon_{i}$ is either an $a$ or a $b$.

For example, one possible ordering would be $a \geq a \geq b \geq b \geq b \geq a$, signifying that after renumbering, $a_{1}(t)$ and $a_{2}(t)$ are always $\geq b_{1}(t), b_{2}(t), b_{3}(t)$, which in turn are $\geq a_{3}(t)$.

(IV) Only the following orderings (if any) can occur:

$$
\begin{aligned}
\text { (i) } a \geq a \geq a \geq b \geq b \geq b \quad \text { (ii) } b \geq b \geq b \geq a \geq a \geq a \\
\text { (iii) } a \geq a \geq b \geq b \geq b \geq a \quad \text { (iv) } b \geq b \geq a \geq a \geq a \geq b \\
\text { (v) } a \geq b \geq b \geq b \geq a \geq a \quad \text { (vi) } b \geq a \geq a \geq a \geq b \geq b .
\end{aligned}
$$

If the ordering is not one of these six, then there would be $i \neq j$ and $k \neq l$ such that $a_{i} \geq b_{k} \geq a_{j} \geq b_{l}$, or else $b_{l} \geq a_{j} \geq b_{k} \geq a_{i}$. But $a_{i}(\tau)=b_{l}(\tau)$ for some $\tau$ and then also $a_{j}(\tau)=a_{i}(\tau)$ contradicting (III).

It suffices by symmetry to consider orderings (i) and (iii). In either case, we define a $b_{j}$ interval to be an interval where $b_{j}=\max \left(b_{1}, b_{2}, b_{3}\right)$ and which is maximal with respect to this property. It is clear that $I_{0}$ is the union of the $b_{j}$ intervals $(j \in\{1,2,3\})$ and (using (II)) that any two have at most an endpoint in common. A common endpoint of a $b_{j}$ and $b_{k}$ interval is clearly 
a point where $b_{j}-b_{k}$ changes sign. Accordingly there is a diagram of $b_{j}$ intervals of the form

$$
\left(\begin{array}{llll}
j_{1} & j_{2} & \ldots & j_{n}
\end{array}\right)
$$

where each $j_{i}$ is either a 1,2 or 3 and $j_{i} \neq j_{i+1}$, signifying that the leftmost interval is a $b_{j_{1}}$ interval, the next a $b_{j_{2}}$ interval, etc., with the rightmost being a $b_{j_{n}}$ interval.

(V) The number $n$ in (28) is $\leq 5$. If $n \geq 4$ then the pattern (28) is one of the following: (12321), (12131), (1213), (1232) (1231) or may be reduced to one of these by renumbering.

The proof may be given diagramatically as follows.

$$
\begin{array}{cccccc} 
& & 1 & 3 & 1 & \text { stuck } \\
& 2 & & & & \\
& & & 1 & \text { stuck } & \\
& 3 & & & \\
& & 2 & 1 & \text { stuck }
\end{array}
$$

Namely, we can always renumber so that the first two intervals are a $b_{1}$ interval followed by a $b_{2}$ interval. The third is then either a $b_{1}$ or a $b_{3}$. If the third is a $b_{1}$ then there can never be another $b_{2}$, since $b_{1}-b_{2}$ has already changed sign twice. Thus the fourth must be a $b_{3}$, the fifth a $b_{1}$, and now $b_{1}-b_{3}$ has also changed sign twice so there is no possibility for a sixth interval. Similarly, if the third interval is a $b_{3}$ then the fourth is a $b_{1}$ or $b_{2}$. In the first case, $b_{1}-b_{2}$ and $b_{1}-b_{3}$ have both changed sign twice so there is no possible fifth interval. In the second case, $b_{2}-b_{3}$ has changed sign twice, so the fifth interval can only be a $b_{1}$, and then $b_{1}-b_{2}$ and $b_{1}-b_{3}$ have also changed sign twice.

(VI) Ordering (i) cannot occur.

Define $a_{i}$ intervals similarly to $b_{j}$ intervals: An $a_{i}$ interval is an interval where $a_{i}=\min \left(a_{1}, a_{2}, a_{3}\right)$ and which is maximal with respect to this property. Of course (V) applies to the $a_{i}$ intervals as well. Observe that in (V) the patterns with length 5 start and end with the same digit. Accordingly, there are at most nine intervals of the form

$$
a_{i} \text { interval } \cap b_{j} \text { interval }
$$

and containing more than one point, and if there are nine such intervals, then the first and last correspond to the same pair $(i, j)$. So at most eight pairs $(i, j)$ actually occur. On the other hand, a point where $a_{i}=b_{j}$ must (by (III)) belong to the interior of an $a_{i}$ interval and a $b_{j}$ interval. Since there are nine choices for $(i, j)$ we have a contradiction.

(VII) Ordering (iii) cannot occur. (This will of course complete the proof of the lemma.) 
We number so that $a_{1} \& a_{2} \geq b_{1} \& b_{2} \& b_{3} \geq a_{3}$. Then we define an $a_{i}$ interval ( $i=1$ or 2 ) to be a maximal interval where $a_{i}=\min \left(a_{1}, a_{2}\right)$. It is easy to see that the pattern of $a_{i}$ intervals is (121), (12) or (1) or can be reduced to one of these by interchanging 1 and 2 .

There are six pairs $(i, j)$ with $i \in\{1,2\}$ and $j \in\{1,2,3\}$. If there are less than four $b_{j}$ intervals, or if there are four and the first and last have the same value of $j$, then we see as in (VI) that for one of these six pairs there is no interval of the form $\left(a_{i}\right.$ interval $\cap b_{j}$ interval $)$ and containing more than one point, which is a contradiction. Thus the pattern of $b_{j}$ intervals may be taken to be either $(21312),(12131),(1213)$ or $(2131)^{4}$. In any of these cases 1 appears in two positions not the first and last. (Namely, the 2nd and 4th positions in the first case, the 1st and 3rd in the second case, etc.) Now there must be a point $\tau$ where $b_{1}(\tau)=a_{3}(\tau)$, hence $b_{1}(\tau)<\min \left(b_{2}(\tau), b_{3}(\tau)\right)$. In moving from $\tau$ to a $b_{1}$ interval, $b_{1}$ must cross both $b_{2}$ and $b_{3}$.

Now consider two cases: Either $\tau$ separates two $b_{1}$ intervals or not. If so, then $b_{1}$ crosses both $b_{2}$ and $b_{3}$ twice between the two $b_{1}$ intervals. Since one of the $b_{1}$ intervals is neither the first nor the last interval, $b_{1}$ must also cross either $b_{2}$ or $b_{3}$ at its opposite endpoint and we have a contradiction. If $\tau$ does not separate two $b_{1}$ intervals then $b_{1}$ nevertheless crosses either $b_{2}$ or $b_{3}$ twice in moving between the two $b_{1}$ intervals, and crosses both $b_{2}$ and $b_{3}$ between $\tau$ and its closest $b_{1}$ interval. This is again a contradiction.

Lemma 3.5. Suppose $\Phi$ satisfies (14) and (15), and $\alpha_{0}, \beta_{0}$ are small enough. Assume that $\left(z_{i}, r_{i}\right) \in \mathbb{R}^{2} \times \mathbb{R}(i=1,2,3)$ are distinct and satisfy $\left|z_{i}\right|+\left|r_{i}\right|<\beta_{0}$. Then there are at most two values of $(z, s)$ with $|z|+|s|<\beta_{0}$ and such that $C(z, s)$ is tangent to the three curves $C\left(z_{i}, r_{i}\right)$ at three different points of $D\left(0, \alpha_{0}\right)$.

Proof. This follows from Lemma 3.4 and (iii) of Lemma 3.3 using appropriate changes of coordinates. Namely, suppose that $C\left(y_{j}, s_{j}\right), j=1,2,3$ are each tangent to each of the curves $C\left(z_{i}, r_{i}\right)$ at three different points as indicated. Since we are working locally we can assume that all the curves in question are graphs. Letting $C\left(z_{i}, r_{i}\right)$ be the graph of $a_{i}$ and $C\left(y_{j}, s_{j}\right)$ the graph of $b_{j}$, we have $n\left(a_{i}-b_{j}\right) \leq 2, n\left(a_{i}-a_{j}\right) \leq 2, n\left(b_{i}-b_{j}\right) \leq 2$, by Lemma 3.3 (iii), and furthermore the tangency assumption implies that $a_{i}-b_{j}$ has a double zero. We conclude by Lemma 3.4 that for some $i_{1}, i_{2}$ and $j$ the tangency point between $C\left(z_{i_{1}}, r_{i_{1}}\right)$ and $C\left(y_{j}, s_{j}\right)$ must coincide with the tangency point between $C\left(z_{i_{2}}, r_{i_{2}}\right)$ and $C\left(y_{j}, s_{j}\right)$, clearly a contradiction.

We now carry out step (2) of the proof of Lemma 3.1(ii). We note to begin with that the points $\xi\left(z_{i}, r_{i}, y, r\right)$ as well as the definition of $\Delta\left(z_{i}, r_{i}, y, r\right)$ depend only on the curve family $C(y, r)=\{x: \Phi(x, y)=r\}$ and not on the

\footnotetext{
${ }^{4}$ It was convenient to renumber some of the cases in (V): (21312) and (2131) are of course equivalent to (12321) and (1232) respectively.
} 
particular choice of defining function $\Phi$. We recall (26): Provided we have appropriate derivative bounds for $\Phi$, there is an estimate

$$
\left|r-\Phi\left(\xi_{i}(y), y\right)\right| \lesssim \epsilon
$$

when $(y, r) \in S$. Here $\xi_{i}(y) \stackrel{\text { def }}{=} \xi\left(z_{i}, r_{i}, y\right)$. We will also repeatedly use $(27)$ which guarantees that the set $C_{\delta}\left(y_{0}, r_{0}\right)$ lies within distance $<<\lambda$ from the point $\xi_{j}\left(y_{0}\right)$.

We let $C_{j}=C\left(z_{j}, r_{j}\right)$, and given a curve $C\left(y_{0}, r_{0}\right)$ we can find numbers $\mu$ and $\nu$ with $\lambda \leq \nu \leq \mu$, and may order the $C_{j}$ 's so that $\left|\xi_{2}\left(y_{0}\right)-\xi_{3}\left(y_{0}\right)\right|=\nu$ and $\left|\xi_{1}\left(y_{0}\right)-\xi_{2}\left(y_{0}\right)\right| \approx \mu,\left|\xi_{1}\left(y_{0}\right)-\xi_{3}\left(y_{0}\right)\right| \approx \mu$. Let $C_{*}$ be a suitable large constant which should be chosen before the constant $A_{1}$ in Lemma 3.1; we note that (30) below is therefore satisfied if $\left|a_{j}-\xi_{j}\left(y_{0}\right)\right|$ is bounded by a fixed multiple of $\frac{\epsilon}{t \lambda}$. We will say that $\Phi$ is $\left(y_{0}, r_{0}\right)$-adapted if there are points $a_{j} \in C_{j}$ such that

$$
\left|a_{j}-\xi_{j}\left(y_{0}\right)\right| \leq C_{*}^{-1} \lambda
$$

and

$$
\begin{aligned}
\Phi\left(a_{1}, y\right) & =0 \\
\nabla_{y} \Phi\left(a_{2}, y\right) & =\left(e \cdot\left(a_{2}-a_{1}\right)\right) \beta
\end{aligned}
$$

for all $y$; here $e$ is the tangent vector to $C_{1}$ at $a_{1}$, and $\beta$ is a vector independent of $y$ with length $\approx 1$.

Lemma 3.6. If $\left(y_{0}, r_{0}\right) \in S$ then a $\left(y_{0}, r_{0}\right)$-adapted defining function will exist (satisfying uniform derivative bounds). Furthermore, it can be taken to be $\left(y_{1}, r_{1}\right)$-adapted for all $\left(y_{1}, r_{1}\right)$ such that $\left|\xi_{j}\left(y_{1}\right)-\xi_{j}\left(y_{0}\right)\right| \leq\left(2 C_{*}\right)^{-1} \lambda$ for all $j$.

Proof. It is easy to see that $\left|e \cdot\left(a_{2}-a_{1}\right)\right| \approx\left|a_{2}-a_{1}\right|$, and we will prove this below (see (36)). We take it as known for now.

Starting from any defining function $\Phi$, we can achieve (31) by replacing $\Phi$ by $\Phi(\cdot, y)-\Phi\left(a_{1}, y\right)$. Assuming this done, we can then achieve (32) by choosing appropriate curvilinear coordinates in the $y$-plane (the "straightening out theorem" in the terminology of [6], applied to the function $\left.\Phi\left(a_{2}, \cdot\right)\right)$, since the hypothesis $(15)$ guarantees that $\left|\nabla_{y} \Phi\left(a_{2}, y\right)\right| \approx\left|a_{2}-a_{1}\right| \approx\left|e \cdot\left(a_{2}-a_{1}\right)\right|$. The last statement follows from the definition of adapted provided we use points with $\left|a_{j}-\xi_{j}\left(y_{0}\right)\right| \leq\left(2 C_{*}\right)^{-1} \lambda$ in the preceding construction.

We fix $\left(y_{0}, r_{0}\right) \in S$ and choose a $\left(y_{0}, r_{0}\right)$-adapted defining function $\Phi$, and we let

$$
T(y)=\left(\begin{array}{cc}
\nabla_{y} \Phi\left(\xi_{1}(y), y\right) & -1 \\
\nabla_{y} \Phi\left(\xi_{2}(y), y\right) & -1 \\
\nabla_{y} \Phi\left(\xi_{3}(y), y\right) & -1
\end{array}\right)
$$


We record the formula for $T(y)^{-1}$ for use below. If $v=\left(v_{1}, v_{2}\right) \in \mathbb{R}^{2}$, then let $v^{*}=\left(-v_{2}, v_{1}\right)$. Then, provided $\operatorname{det} T(y) \neq 0, T(y)^{-1}$ is equal to

$$
(\operatorname{det} T(y))^{-1}\left(\begin{array}{cc}
\left(\nabla_{y} \Phi\left(\xi_{2}(y), y\right)-\nabla_{y} \Phi\left(\xi_{3}(y), y\right)\right)^{*} & \nabla_{y} \Phi\left(\xi_{2}(y), y\right) \wedge \nabla_{y} \Phi\left(\xi_{3}(y), y\right) \\
\left(\nabla_{y} \Phi\left(\xi_{3}(y), y\right)-\nabla_{y} \Phi\left(\xi_{1}(y), y\right)\right)^{*} & \nabla_{y} \Phi\left(\xi_{3}(y), y\right) \wedge \nabla_{y} \Phi\left(\xi_{1}(y), y\right) \\
\left(\nabla_{y} \Phi\left(\xi_{1}(y), y\right)-\nabla_{y} \Phi\left(\xi_{2}(y), y\right)\right)^{*} & \nabla_{y} \Phi\left(\xi_{1}(y), y\right) \wedge \nabla_{y} \Phi\left(\xi_{2}(y), y\right)
\end{array}\right)^{t}
$$

where $T^{t}$ means transpose of $T$. We also define $s_{j}=\Phi\left(\xi_{j}\left(y_{0}\right), y_{0}\right)$, and note that $\left|s_{j}-r_{0}\right| \lesssim \epsilon$ by $(29)$.

Lemma 3.7. $\left|\operatorname{det} T\left(y_{0}\right)\right| \approx \mu^{2} \nu$ and $\left\|T\left(y_{0}\right)^{-1}\right\| \lesssim(\mu \nu)^{-1}$.

Proof. If $y=y_{0}$ then the entries of the cofactor matrix in (33) are clearly $\lesssim \mu$ and it therefore suffices to show that $\left|\operatorname{det} T\left(y_{0}\right)\right| \approx \mu^{2} \nu$. However, we have

$$
\left|\Phi\left(\xi_{i}\left(y_{0}\right), y_{0}\right)-\Phi\left(\xi_{j}\left(y_{0}\right), y_{0}\right)\right| \lesssim \epsilon
$$

so by (14) there are points $x_{1}, x_{2}, x_{3} \in \mathbb{R}^{2}$ with $\left|x_{i}-\xi_{i}\left(y_{0}\right)\right| \lesssim \epsilon$ and $\Phi\left(x_{1}, y_{0}\right)=\Phi\left(x_{2}, y_{0}\right)=\Phi\left(x_{3}, y_{0}\right)$. Note that then also

$$
\left|x_{i}-x_{j}\right| \approx\left|\xi_{i}\left(y_{0}\right)-\xi_{j}\left(y_{0}\right)\right|
$$

since $\epsilon$ is small compared with $\lambda$. Define

$$
M=\left(\begin{array}{cc}
\nabla_{y} \Phi\left(x_{1}, y_{0}\right) & -1 \\
\nabla_{y} \Phi\left(x_{2}, y_{0}\right) & -1 \\
\nabla_{y} \Phi\left(x_{3}, y_{0}\right) & -1
\end{array}\right)
$$

Then Lemma 3.2 implies that

$$
\begin{aligned}
|\operatorname{det} M| & \approx\left|x_{1}-x_{2}\right|\left|x_{2}-x_{3}\right|\left|x_{3}-x_{1}\right| \\
& \approx \mu^{2} \nu
\end{aligned}
$$

where the last line follows from (34). On the other hand, $\mid \operatorname{det}\left(T\left(y_{0}\right)\right)-$ $\operatorname{det}(M) \mid \lesssim \epsilon(\mu+\epsilon)$ since $\left|\nabla_{y} \Phi\left(\xi_{i}\left(y_{0}\right), y_{0}\right)-\nabla_{y} \Phi\left(x_{i}, y_{0}\right)\right| \lesssim \epsilon$ and the cofactors of $T\left(y_{0}\right)$ are $\lesssim \mu$. We know that $\epsilon \ll \lambda^{2}$, so $\epsilon(\mu+\epsilon)$ is small compared with $\mu^{2} \nu$. This completes the proof.

Lemma 3.8. If $y$ is such that $\left|T\left(y_{0}\right)\left(y-y_{0}, r-r_{0}\right)\right|<\epsilon$ for some $r$ then

$$
T(y) T\left(y_{0}\right)^{-1}=I+E(y)
$$

where $I$ is the identity matrix and $E(y)$ is a matrix with norm $\leq \frac{1}{100}$.

Proof. We can assume that $a_{1}$ is the origin (thus by (30), all the points $\xi_{j}\left(y_{0}\right)$ are at distance $\lesssim \mu$ from the origin) and furthermore that the unit tangent vector to $C_{1}$ at the origin is parallel to the $x^{(1)}$ axis.

Let $e_{j}(x)$ be the unit tangent vector to $C_{j}$ at the point $x \in C_{j}$. We claim first that if $x \in C_{j}$ for some $j$ and $|x| \leq \mu$, then

$$
\left|x^{(2)}\right| \lesssim \mu^{2}
$$




$$
\left|e_{j}(x)^{(2)}\right| \lesssim \mu \text {. }
$$

We will repeatedly use the following simple fact: If $C$ is a smooth curve and there is one point $x \in C$ with $|x| \lesssim \mu$ such that $\left|x^{(2)}\right| \lesssim \mu^{2}$ and the $x^{(2)}$ component of the unit tangent vector to $C$ at $x$ is $\lesssim \mu$, then the same properties hold at every point of $C$ which is at distance $\lesssim \mu$ from the origin.

In particular, $\left(3 \overline{5)}\right.$ and $(36)$ are obvious if $j=1$, since $C_{1}$ passes through the origin and has a horizontal tangent vector there. If $j=2$ or 3 , then set $x=\xi_{j}\left(y_{0}\right) \in C_{j} \cap C\left(y_{0}, s_{j}\right)$. We have $\left|s_{j}-s_{1}\right| \lesssim \epsilon$, so by (14) we can find a point $\tilde{x} \in C\left(y_{0}, s_{1}\right)$ which is at distance $\lesssim \epsilon$ from $\xi_{j}\left(r_{0}\right)$. Let $\tilde{e}$ be the unit tangent vector to $C\left(y_{0}, s_{1}\right)$ at $\tilde{x}$. Clearly then $|\tilde{x}| \lesssim \mu$. $C\left(y_{0}, s_{1}\right)$ is tangent to $C_{1}$ at $\xi_{1}\left(y_{0}\right)$, which is within $\mu$ of the origin, and it follows that $\left|\tilde{x}^{(2)}\right| \lesssim \mu^{2}$ and $\left|\tilde{e}^{(2)}\right| \lesssim \mu$. Hence $\left|x^{(2)}\right| \lesssim \mu^{2}+\epsilon \lesssim \mu^{2}$. On the other hand $e_{j}(x)$ coincides with the unit tangent vector to $C\left(y_{0}, s_{j}\right)$ at $x$ and therefore $\left|e_{j}(x)-\tilde{e}\right| \lesssim \epsilon$, so $\left|e_{j}(x)^{(2)}\right| \lesssim \mu$. This proves (35) and (36). We note that if $x, \bar{x} \in C_{j}$ and $|x|+|\bar{x}| \lesssim \mu$, then (36) implies

$$
\left|x^{(2)}-\bar{x}^{(2)}\right| \lesssim \mu|x-\bar{x}| \text {. }
$$

Next, we claim that if $|x| \lesssim \mu$ and $\left|x^{(2)}\right| \lesssim \mu^{2}$ then

$$
\frac{d}{d x^{(1)}} \nabla_{y} \Phi(x, y)=\beta+\mathcal{O}(\mu) \text {. }
$$

We start by proving (38) when $x=0$. We expand $\nabla_{y} \Phi(\cdot, y)$ in a Taylor series with remainder:

$$
\begin{aligned}
\nabla_{y} \Phi(x, y) & =\nabla_{y} \Phi(0, y)+x^{(1)} \frac{\partial \nabla_{y} \Phi}{\partial x^{(1)}}(0, y)+\mathcal{O}\left(x^{(2)}+\left(x^{(1)}\right)^{2}\right) \\
& =x^{(1)} \frac{\partial \nabla_{y} \Phi}{\partial x^{(1)}}(0, y)+\mathcal{O}\left(x^{(2)}+\left(x^{(1)}\right)^{2}\right)
\end{aligned}
$$

using (31). Now we set $x=a_{2}$ and use (32):

$$
\begin{aligned}
a_{2}{ }^{(1)} \beta=\nabla_{y} \Phi\left(a_{2}, y\right) & =a_{2}{ }^{(1)} \frac{\partial \nabla_{y} \Phi}{\partial x^{(1)}}(0, y)+a_{2}{ }^{(2)} \frac{\partial \nabla_{y} \Phi}{\partial x^{(2)}}(0, y)+\mathcal{O}\left(\left|a_{2}\right|^{2}\right) \\
& =a_{2}{ }^{(1)} \frac{\partial \Phi}{\partial x^{(1)}}(0, y)+\mathcal{O}\left(\mu^{2}\right)
\end{aligned}
$$

by (35). This proves (38) when $x=0$. It follows that (38) holds for any $x$ with $|x| \leq \mu$.

An immediate consequence of (38) is that if $|x|+|\bar{x}| \lesssim \mu$ and $\left|x^{(2)}\right|+$ $\left|\bar{x}^{(2)}\right| \lesssim \mu^{2}$ then

$$
\nabla_{y} \Phi(x, y)-\nabla_{y} \Phi(\bar{x}, y)=(x-\bar{x})^{1} \beta+\mathcal{O}\left(\mu^{2}\right) .
$$

We now prove two more bounds of the same type. First we claim that if $x, \bar{x} \in C_{j}$ and $|x|+|\bar{x}| \lesssim \mu$, then

$$
\nabla_{y} \Phi(x, y)-\nabla_{y} \Phi(\bar{x}, y)=(x-\bar{x})^{(1)} \beta+\mathcal{O}(\mu|x-\bar{x}|) .
$$


This follows from the preceding estimates and the mean value theorem: Let $\gamma$ be the line segment connecting $x$ and $\bar{x}$, then

$$
\begin{aligned}
& \left|\left(\nabla_{y} \Phi(x, y)-x^{(1)} \beta\right)-\left(\nabla_{y} \Phi(\bar{x}, y)-\bar{x}^{(1)} \beta\right)\right| \\
& \lesssim\left|x^{(1)}-\bar{x}^{(1)}\right| \max _{u \in \gamma}\left|\frac{d}{d x^{(1)}} \nabla_{y} \Phi(u, y)-\beta\right|+\left|x^{(2)}-\bar{x}^{(2)}\right| \max _{u \in \gamma}\left|\frac{d}{d x^{(2)}} \nabla_{y} \Phi(u, y)\right| \\
& \lesssim|x-\bar{x}| \cdot \mu+\mu|x-\bar{x}| \cdot 1
\end{aligned}
$$

by (38) and (37). This proves (41).

Next, we claim that if $|x| \lesssim \mu$ and $\left|x^{(2)}\right| \lesssim \mu$, then

$$
\left|\nabla_{y}^{2} \Phi(x, y)\right| \lesssim \mu^{2}
$$

Namely, let $e$ be the unit vector in the direction from 0 to $a_{2}$. Fix $i$ and $j$ and let $f(x)=\nabla_{y}^{2} \Phi(x, y)$. By (31) and (32), $f$ is a smooth function vanishing at 0 and $a_{2}$ and therefore $|e \cdot \nabla f(0)| \lesssim \mu$. The $x^{(2)}$ component of $e$ is $\lesssim \mu$ by (35) so we may conclude that $\left|\frac{\partial f}{\partial x^{(1)}}(0)\right| \lesssim \mu$, and therefore $\left|\frac{\partial f}{\partial x^{(1)}}\right| \lesssim \mu$ at all points within $\mu$ of the origin. Estimate (42) now follows from the mean value theorem and: $|f(x)|=|f(x)-f(0)| \lesssim \mu\left|x^{(1)}-a_{i}{ }^{(1)}\right|+\left|x^{(2)}\right| \lesssim \mu^{2}$.

Now suppose that $(y, r) \in \mathbb{R}^{2} \times \mathbb{R}$ is such that $\left|T\left(y_{0}\right)\left(y-y_{0}, r-r_{0}\right)\right|<\epsilon$. Then we claim that

$$
\begin{aligned}
\left|y-y_{0}\right| & \lesssim \frac{\epsilon}{\mu \nu} \\
\left|\beta \cdot\left(y-y_{0}\right)\right| & \lesssim \frac{\epsilon}{\nu} \\
\left|\xi_{j}(y)-\xi_{j}\left(y_{0}\right)\right| & \lesssim \frac{\epsilon}{t \nu} .
\end{aligned}
$$

Namely, (43) follows immediately from Lemma 3.7. Furthermore, by (40) we have $\nabla_{y} \Phi\left(\xi_{2}\left(y_{0}\right), y_{0}\right)-\nabla_{y} \Phi\left(\xi_{1}\left(y_{0}\right), y_{0}\right)=\left(\xi_{2}\left(y_{0}\right)-\xi_{1}\left(y_{0}\right)\right)^{(1)} \beta+$ $\mathcal{O}\left(\mu^{2}\right)$, with $\left|\left(\xi_{2}\left(y_{0}\right)-\xi_{1}\left(y_{0}\right)\right)^{(1)}\right| \approx \mu$. The quantity $\left(\nabla_{y} \Phi\left(\xi_{2}\left(y_{0}\right), y_{0}\right)-\right.$ $\left.\nabla_{y} \Phi\left(\xi_{1}\left(y_{0}\right), y_{0}\right)\right) \cdot\left(y-y_{0}\right)$ is the difference between the second and first components of $T\left(y_{0}\right)\left(y-y_{0}, r-r_{0}\right)$ and is therefore $\leq 2 \epsilon$, so $\left|\beta \cdot\left(y-y_{0}\right)\right| \lesssim$ $\frac{\epsilon}{\mu}+\mu\left|y-y_{0}\right| \lesssim \frac{\epsilon}{\nu}$ (by (43)), which is (44).

In order to prove (45) we consider the variation $y(s)=y_{0}+s\left(y-y_{0}\right), 0 \leq$ $s \leq 1$. We will denote the $s$-derivative of the function $f$ by $\dot{f}$. Recall that $e_{j}(x), x \in C_{j}$, is the unit tangent vector field to $C_{j}$. Assume temporarily that $\left|\xi_{j}(y(s))-\xi_{j}(y(0))\right| \leq \mu$ for all $s$. Consider the equation

$$
e_{j}\left(\xi_{j}(s)\right) \cdot \nabla_{x} \Phi\left(\xi_{j}(y(s)), y(s)\right)
$$

which defines $\xi_{j}(s)$ implicitly. Differentiate it with respect to $s$ and take the component of the resulting equation in the direction $e_{j}\left(\xi_{j}(s)\right)$. This gives

$$
\dot{\xi}_{j}=\frac{\left(e_{j} \cdot \nabla_{x}\right) \nabla_{y} \Phi\left(\xi_{j}(y(s)), y(s)\right) \cdot\left(y-y_{0}\right)}{\left(e_{j} \cdot \nabla_{x}\right)^{2} \Phi\left(\xi_{j}(y(s)), y(s)\right)} .
$$


The expression in the denominator is $\gtrsim\left|y(s)-z_{j}\right|$ by the curvature hypothesis (15). Since $\left|y(s)-y_{0}\right| \leq \frac{\epsilon}{\mu \nu} \ll t$, we conclude that $\left|\left(e \cdot \nabla_{x}\right)^{2} \Phi(\xi, y)\right| \gtrsim t$. On the other hand, the $x^{(2)}$-component of $e_{j}$ is $\lesssim \mu$ and therefore

$$
\begin{aligned}
& \left(e_{j} \cdot \nabla_{x}\right) \nabla_{y} \Phi\left(\xi_{j}(y(s)), y(s)\right) \cdot\left(y-y_{0}\right) \\
& =e^{(1)} \frac{d}{d x^{(1)}} \nabla_{y} \Phi\left(\xi_{j}(y(s)), y(s)\right) \cdot\left(y-y_{0}\right)+\mathcal{O}\left(\mu\left|y-y_{0}\right|\right) \\
& =e^{(1)} \beta \cdot\left(y-y_{0}\right)+\mathcal{O}\left(\mu\left|y-y_{0}\right|\right) \quad \text { by }(38) \\
& \lesssim \frac{\epsilon}{\nu} \quad \text { by }(43) \text { and }(44) .
\end{aligned}
$$

We conclude that $\left|\dot{\xi}_{j}\right| \lesssim \frac{\epsilon}{t \nu}$. Thus we have shown that under the a priori assumption on $v$ that $\left|\xi_{j}(s)-\xi_{j}(0)\right| \leq \mu$ for all $s$ we actually have $\mid \xi_{j}(s)-$ $\xi_{j}(0) \mid \leq C \frac{\epsilon}{t \nu}$ for all $s$. Since $\frac{\epsilon}{t \nu}$ is small compared with $\mu$ it follows that the a priori assumption can be dropped, i.e., (45) holds.

Next we claim that if $\left|T\left(y_{0}\right)\left(y-y_{0}, r-r_{0}\right)\right|<\epsilon$ then

$$
\begin{aligned}
\left|\nabla_{y} \Phi\left(\xi_{j}(y), y\right)-\nabla_{y} \Phi\left(\xi_{j}\left(y_{0}\right), y_{0}\right)\right| & \lesssim \frac{\epsilon}{t \nu} \\
\left|\left(\nabla_{y} \Phi\left(\xi_{j}(y), y\right)-\nabla_{y} \Phi\left(\xi_{j}\left(y_{0}\right), y_{0}\right)\right) \wedge \beta\right| & \lesssim \mu \frac{\epsilon}{t \nu} .
\end{aligned}
$$

For the proof we write

$$
\begin{aligned}
& \nabla_{y} \Phi\left(\xi_{j}(y), y\right)-\nabla_{y} \Phi\left(\xi_{j}\left(y_{0}\right), y_{0}\right) \\
& =\left(\nabla_{y} \Phi\left(\xi_{j}(y), y\right)-\nabla_{y} \Phi\left(\xi_{j}\left(y_{0}\right), y\right)\right)+\left(\nabla_{y} \Phi\left(\xi_{j}\left(y_{0}\right), y\right)-\nabla_{y} \Phi\left(\xi_{j}\left(y_{0}\right), y_{0}\right)\right) \\
& \stackrel{\text { def }}{=} \mathrm{I}+\mathrm{II}
\end{aligned}
$$

and make the following estimates:

$$
|\mathrm{II}| \lesssim \mu^{2} \cdot \frac{\epsilon}{\mu \nu}=\mu \frac{\epsilon}{\nu} \leq \mu \frac{\epsilon}{t \nu}
$$

by (42) and (43). Also

$$
|\mathrm{I}| \lesssim \frac{\epsilon}{t \nu}
$$

by (45), and finally

$$
|\mathrm{I} \wedge \beta| \lesssim \mu \frac{\epsilon}{t \nu}
$$

by (45) and (41). This proves (46) and (47).

By (40), which is applicable by (45) and (35), we have

$$
\nabla_{y} \Phi\left(\xi_{j}\left(y_{0}\right), y_{0}\right)-\nabla_{y} \Phi\left(\xi_{k}\left(y_{0}\right), y_{0}\right)=\left(\xi_{j}\left(y_{0}\right)-\xi_{k}\left(y_{0}\right)\right)^{(1)} \beta+\mathcal{O}\left(\mu^{2}\right)
$$


with $\left|\xi_{j}\left(y_{0}\right)-\xi_{k}\left(y_{0}\right)\right| \lesssim \mu$. It follows using (46) and (47) that

$$
\begin{aligned}
& \left|\left(\nabla_{y} \Phi\left(\xi_{i}(y), y\right)-\nabla_{y} \Phi\left(\xi_{i}\left(y_{0}\right), y_{0}\right)\right) \wedge\left(\nabla_{y} \Phi\left(\xi_{j}\left(y_{0}\right), y_{0}\right)-\nabla_{y} \Phi\left(\xi_{k}\left(y_{0}\right), y_{0}\right)\right)\right| \\
& \lesssim \mu^{2} \frac{\epsilon}{t \nu}
\end{aligned}
$$

for all $i, j$ and $k$.

We now prove Lemma 3.8. Namely, if $v=\left(v_{1}, v_{2}\right) \in \mathbb{R}^{2}$ then, using (33), each component of $\left(T(y)-T\left(y_{0}\right)\right) T\left(y_{0}\right)^{-1}$ is of the form

$$
\begin{aligned}
\left(\operatorname{det} T\left(y_{0}\right)\right)^{-1}\left(\nabla_{y} \Phi\left(\xi_{i}(y), y\right)-\nabla_{y} \Phi\right. & \left.\left(\xi_{i}\left(y_{0}\right), y_{0}\right)\right) \\
& \wedge\left(\nabla_{y} \Phi\left(\xi_{j}\left(y_{0}\right), y_{0}\right)-\nabla_{y} \Phi\left(\xi_{k}\left(y_{0}\right), y_{0}\right)\right)
\end{aligned}
$$

for some $i, j, k$. We have $\left|\operatorname{det} T\left(y_{0}\right)\right| \approx \mu^{2} \nu$ by Lemma 3.7 and we therefore conclude by (48) that $\left\|(T(y)-T(0)) T(0)^{-1}\right\| \lesssim \frac{\epsilon}{t \nu^{2}}$, which is small. The lemma follows.

We now define

$$
G(y, r)=\left(\begin{array}{l}
\Phi\left(\xi_{1}(y), y\right)-r \\
\Phi\left(\xi_{2}(y), y\right)-r \\
\Phi\left(\xi_{3}(y), y\right)-r
\end{array}\right) .
$$

We let $C_{0}$ be a suitable constant and $E(y, r)=(y, r)+D G(y, r)^{-1} D\left(0, C_{0} \epsilon\right)$, i.e., $E(y, r)$ is the ellipsoid centered at $(y, r)$ which the derivative of $G$ at $(y, r)$ maps onto the disc $D\left(C_{0}, \epsilon\right)$.

Lemma 3.9. (i) $E\left(y_{0}, r_{0}\right)$ has volume $\approx \frac{\epsilon^{3}}{\mu^{2} \nu}$ and diameter $\lesssim \frac{\epsilon}{\mu \nu}$

(ii) $G$ defines a diffeomorphism from a subset of $E\left(y_{0}, r_{0}\right)$ onto $D(0, \epsilon)$.

(iii) If $(y, r) \in E\left(y_{0}, r_{0}\right)$ then $E\left(y_{0}, r_{0}\right)$ and $E(y, r)$ are comparable ellipsoids, i.e., each is contained in the dilation of the other around its center by a factor $C_{1}$.

Proof. Observe that $D G(y, r)=T(y)$, since $\nabla_{x} \Phi\left(\xi_{i}(y), y\right)$ is parallel to $\nabla_{x} \Phi\left(\xi_{i}(y), z_{i}\right)$ and therefore perpendicular to any column of $d \xi_{i}(y)$.

Part (i) is immediate from Lemma 3.7. For (iii), we use that matrices $A$ and $B$ such that $\left\|A^{-1} B-I\right\| \leq \frac{1}{2}$ map any fixed disc centered at the origin to comparable ellipsoids. If we set $A=T(y)^{-1}, B=T\left(y_{0}\right)^{-1}$ then $\left\|A^{-1} B-I\right\| \leq \frac{1}{2}$ by Lemma 3.8 and (iii) follows.

To prove (ii), we use the following version of the inverse function theorem: If $\Omega \subset \mathbb{R}^{3}$ is open, $f: \Omega \rightarrow \mathbb{R}^{3}, f(a)=b$ and $|D f(x)-I| \leq \frac{1}{100}$ when $x \in D\left(0, C_{2} \epsilon\right)$, then $f$ maps some subset of $D\left(a, C_{2} \epsilon\right)$ diffeomorphically onto $D(b, \epsilon)$. This follows from the usual proof of the IFT: We can assume $a=b=0$. Then with the stated hypotheses, if $y \in D(0, \epsilon)$ then the map $x \rightarrow x-f(x)+y$ will be a contraction mapping from $D\left(0, C_{2} \epsilon\right)$ into itself, etc. 
We apply this version of the IFT to the map $G \circ D G\left(y_{0}, r_{0}\right)^{-1}$, which satisfies the hypothesis in view of Lemma 3.8 ( $\epsilon$ in Lemma 3.8 can clearly be replaced by $\left.C_{2} \epsilon\right)$. The result follows.

We may now finish the proof of Lemma 3.1 as follows. Suppose that $\left(y_{0}, r_{0}\right) \in S$ is given and choose an adapted defining function. Then by (ii) of Lemma 3.9 one of the two points $(\eta, \rho)$ must belong to $E\left(y_{0}, r_{0}\right)$. Also $\left|\xi_{j}\left(y_{0}\right)-\xi_{j}(\eta)\right|$ is small compared with $\lambda$ by (45). It follows therefore that there is a defining function $\Phi$ which is $\left(y_{0}, r_{0}\right)$ - adapted for all $\left(y_{0}, r_{0}\right)$ with a given $(\eta, \rho)$. Using this $\Phi$, it follows by (iii) of Lemma 3.9 that each point $\left(y_{0}, r_{0}\right)$ must belong to a fixed dilation of $E(\eta, \rho)$, and the proof is complete. The final statement (19) follows since the projection of each ellipsoid $E(\eta, \rho)$ on any given axis will be an interval of length $\lesssim \frac{\epsilon}{\lambda^{2}}$.

\section{Proof of Theorem $2^{\prime}$, part 2.}

We will prove the following general version of Theorem $2^{\prime}$.

Theorem 4.1. Let $\Phi: U \rightarrow \mathbb{R}$ be a function satisfying hypotheses (14), (15), with $\Phi(a, b)=r_{0}$, and let $\epsilon_{0}$ be a small constant. If $\delta>0$ is small and $f: \mathbb{R}^{2} \rightarrow \mathbb{R}$, supp $f \subset D\left(a, \epsilon_{0}\right)$ then define $M_{\delta} f:\left(r_{0}-\epsilon_{0}, r_{0}+\epsilon_{0}\right) \rightarrow \mathbb{R}$ via

$$
M_{\delta} f(r)=\sup _{y \in D\left(b, \epsilon_{0}\right)} \delta^{-1} \int_{C_{\delta}(y, r)}|f| .
$$

Then

$$
\left\|M_{\delta} f\right\|_{q} \lesssim \delta^{-\frac{1}{2}\left(\frac{3}{p}-1\right)}\|f\|_{p}, \quad p<\frac{8}{3}, q=2 p^{\prime} .
$$

The $p=1, q=\infty$ case of Theorem 4.1 is trivial, and it therefore suffices to prove the following restricted weak type bound:

$$
\left|\left\{r \in\left[\frac{1}{2}, 2\right]: M_{\delta} \chi_{E}(r)>\lambda\right\}\right| \leq C\left(\frac{|E|}{\delta^{\frac{1}{6}} \lambda^{\frac{8}{3}}}\right)^{\frac{6}{5}} .
$$

Here the set $E$ is contained in $D\left(0, \epsilon_{0}\right) \subset \mathbb{R}^{2}$. As usual it is convenient to discretize; since we can estimate the measure of a set in terms of its $\delta$-entropy it suffices to prove the following.

Assume there are $M 3 \delta$-separated values $r_{j} \in\left[r_{0}-\epsilon_{o}, r_{0}+\epsilon_{0}\right]$ and points $x_{j} \in D\left(0, \epsilon_{0}\right)$ such that $\left|E \cap C_{\delta}\left(x_{j}, r_{j}\right)\right| \geq \lambda\left|C_{\delta}\left(x_{j}, r_{j}\right)\right|$. Then

$$
M_{\delta} \leq C\left(\frac{|E|}{\delta^{\frac{1}{6}} \lambda^{\frac{8}{3}}}\right)^{\frac{6}{5}} .
$$

We can assume that $M$ is large; for $M$ smaller than any fixed constant (51) holds because $M \neq 0$ implies $|E| \gtrsim \lambda \delta$. We may also assume that $\lambda \leq 1$. 
To prove (51) we let $\mu$ ("multiplicity") be the smallest number with the following property: There are at least $\frac{M}{2}$ values of $j$ such that

$$
\left|E \cap C_{\delta}\left(x_{j}, r_{j}\right) \cap\left\{x: \operatorname{card}\left(\left\{i: x \in C_{\delta}\left(x_{i}, r_{i}\right)\right\}\right) \leq \mu\right\}\right| \geq \frac{\lambda}{2}\left|C_{\delta}\left(x_{j}, r_{j}\right)\right| .
$$

The main estimate is

$$
\mu \lesssim M^{\frac{1}{6}} \lambda^{-\frac{5}{3}}
$$

Before proving (53) we introduce some more notation, as follows. For any $t \in[\delta, 1]$ and $\epsilon \in[\delta, 1]$, let

$$
a(t, \epsilon)=C_{1}^{-1}\left(\frac{\delta}{\epsilon}\right)^{\alpha}\left(\frac{M \delta}{t}+\frac{t}{M \delta}\right)^{-\alpha} .
$$

Here $\alpha$ is a sufficiently small positive constant, and $C_{1}$ is a positive constant (easily shown to exist) which is large enough that

$$
\sum_{\substack{k \geq 0 \\ l \geq 0}} a\left(2^{k} \delta, 2^{l} \delta\right)<1
$$

for all $M$ and $\delta$. Also let

$$
\begin{aligned}
\bar{\lambda}(t, \epsilon) & =a(t, \epsilon) \frac{\lambda}{2} \\
\bar{\mu}(t, \epsilon) & =a(t, \epsilon) \mu \\
\bar{M}(t, \epsilon) & =a(t, \epsilon) \frac{M}{2}
\end{aligned}
$$

Let $\Delta(z, s, y, r)$ be the function of Lemma 3.1, and for $i, j \in\{1, \ldots, M\}$ define a number $\Delta_{i j}$ as follows:

$$
\Delta_{i j}=\max \left(\delta, \Delta\left(x_{i}, r_{i}, x_{j}, r_{j}\right)\right) .
$$

For each $j \in\{1, \ldots, M\}, t \in[\delta, 1], \epsilon \in[\delta, 1]$, let

$$
\begin{aligned}
S_{t, \epsilon}\left(x_{j}, r_{j}\right) \stackrel{\text { def }}{=} & \left\{i: C_{\delta}\left(x_{j}, r_{j}\right) \cap C_{\delta}\left(x_{i}, r_{i}\right) \neq \emptyset, t \leq\left|r_{i}-r_{j}\right|+\left|x_{i}-x_{j}\right| \leq 2 t\right. \\
& \text { and } \left.\epsilon \leq \Delta_{i j} \leq 2 \epsilon\right\} \\
A_{t, \epsilon}\left(x_{j}, r_{j}\right) \stackrel{\text { def }}{=} & \left\{x \in C_{\delta}\left(x_{j}, r_{j}\right): \operatorname{card}\left(\left\{i \in S_{t, \epsilon}\left(x_{j}, r_{j}\right): x \in C_{\delta}\left(x_{i}, r_{i}\right)\right\}\right)\right. \\
& \geq \bar{\mu}(t, \epsilon)\} .
\end{aligned}
$$

Lemma 4.1. There are numbers $t \in[\delta, 1]$ and $\epsilon \in[\delta, 1]$ with the following property:

There are $\geq \bar{M}(t, \epsilon)$ values of $j$ such that $\left|A_{t \epsilon}\left(x_{j}, r_{j}\right)\right| \geq \bar{\lambda}(t, \epsilon)\left|C_{\delta}\left(x_{j}, r_{j}\right)\right|$. 
Proof. This is a routine pigeonhole argument. By the minimality of $\mu$ there are at least $\frac{M}{2}$ values of $j$ such that $\left|\tilde{E}_{j}\right| \geq \frac{\lambda}{2}\left|C_{\delta}\left(x_{j}, r_{j}\right)\right|$ where

$$
\tilde{E}_{j}=E \cap C_{\delta}\left(x_{j}, r_{j}\right) \cap\left\{x: \operatorname{card}\left(\left\{i: x \in C_{\delta}\left(x_{i}, r_{i}\right)\right\}\right) \geq \mu\right\} .
$$

For any such $j$ and any $x \in \tilde{E}_{j},(54)$ implies there are $t=2^{k} \delta$ and $\epsilon=2^{l} \delta$ such that $x \in A_{t \epsilon}\left(x_{j}, r_{j}\right)$. Consequently, using (54) again, for any such $j$ there are $t=2^{k} \delta$ and $\epsilon=2^{l} \delta$ such that

$$
\left|A_{t \epsilon}\left(x_{j}, r_{j}\right)\right| \geq \bar{\lambda}(t, \epsilon)\left|C_{\delta}\left(x_{j}, r_{j}\right)\right| .
$$

By (54) once more, there must be a choice of $t$ and $\epsilon$ such that (56) holds for at least $\bar{M}(t, \epsilon)$ values of $j$. This finishes the proof.

We also want to note the following simple fact which will be used in a crucial way below.

Lemma 4.2. Let $\phi$ be a smooth function on a disc $D(0, r) \subset \mathbb{R}^{2}$, with $\nabla \phi \neq 0$ and $\phi(0)=0$. Let $\Gamma^{\delta}=\left\{x \in D\left(0, \frac{r}{2}\right):|\phi(x)|<\delta\right\}$. Suppose $F \subset \Gamma^{\delta}$. Then there are three subsets $F_{1}, F_{2}, F_{3} \subset F$ such that (i) $\left|F_{i}\right| \geq C^{-1}|F|$, $i=1,2,3$ and (ii) $\operatorname{dist}\left(F_{i}, F_{j}\right) \geq(C \delta)^{-1}|F|$ if $i \neq j$.

The constant $C$ of course depends only on bounds for derivatives of $\phi$, a lower bound for $|\nabla \phi|$, and $r$.

Proof of (53). We may assume by a partition of unity and change of variable that $\phi(x)=x_{(2)}$ and $r=1$.

We partition $\left(-\frac{1}{2}, \frac{1}{2}\right.$ ) into five subintervals $I_{1}, \cdots, I_{5}$ (numbered from left to right) in such a way that $\left|\left\{x \in F: x_{(1)} \in I_{k}\right\}\right|$ is independent of $k$, and is therefore equal to $\frac{|F|}{5}$. Set $F_{j}=\left\{x \in F: x_{(1)} \in I_{2 j-1}\right\}$. It is clear that property (i) then holds. To prove (ii), note that for $k=2$ or 4 , $\left|I_{k}\right| \geq \frac{\left|\left\{x \in F: x_{(1)} \in I_{j}\right\}\right|}{2 \delta}=\frac{|F|}{10 \delta}$. Consequently $\operatorname{dist}\left(F_{i}, F_{j}\right) \geq \frac{|F|}{10 \delta}$, as claimed.

We split the proof of (53) into two cases.

(i) $\bar{\lambda} \geq C_{2} \sqrt{\frac{\epsilon}{t}}$

(ii) $\bar{\lambda} \leq C_{2} \sqrt{\frac{\epsilon}{t}}$

where $C_{2}$ is a sufficiently large constant.

In case (i), which is the main case, we let $S$ be the set of $M$ "tubes" in (51), and let $\bar{S}$ be the set of at least $\bar{M}$ tubes in Lemma 4.1. Let $Q$ be the set of all quadruples $\left(j, j_{1}, j_{2}, j_{3}\right)$ with $C\left(x_{j}, r_{j}\right) \in \bar{S}, C\left(x_{j_{i}}, r_{j_{i}}\right) \in S$ for $i=1,2,3$ and such that $j_{i} \in S_{t, \epsilon}\left(x_{j}, r_{j}\right)$ for each $i \in\{1,2,3\}$ and furthermore the distance between any two of the three sets $C_{\delta}\left(x_{j}, r_{j}\right) \cap C_{\delta}\left(x_{j_{i}}, r_{j_{i}}\right)$ is at least $C_{3}^{-1} \bar{\lambda}$. Here $C_{3}$ is a suitable constant which should be chosen before $C_{2}$. 
We will make two different estimates on the cardinality of $Q$. On the one hand, if $C_{2}$ is large enough then by $(19)^{5}$, for any fixed $j_{1}, j_{2}, j_{3}$ there are $\lesssim \frac{\epsilon}{\delta} \bar{\lambda}^{-2}$ values of $j$ such that $\left(j, j_{1}, j_{2}, j_{3}\right) \in Q$. Also it follows from the definition of $Q$ that there are $\lesssim M \min \left(M, \frac{t}{\delta}\right)^{2}$ possible choices for $\left(j_{1}, j_{2}, j_{3}\right)$ : There are at most $M$ choices for $j_{1}$, and once $j_{1}$ is fixed there are $\lesssim \min \left(M, \frac{t}{\delta}\right)$ possibilities for each of $j_{2}$ and $j_{3}$, since $\left|r_{j_{1}}-r_{j_{i}}\right| \leq$ $\left|r_{j_{1}}-r_{j}\right|+\left|r_{j}-r_{j_{i}}\right| \leq 4 t$ for $i=2$ or 3 . We conclude that

$$
\operatorname{card} Q \lesssim \frac{\epsilon}{\delta} \bar{\lambda}^{-2} M \min \left(M, \frac{t}{\delta}\right)^{2} .
$$

On the other hand, if we fix $j$ with $C\left(x_{j}, r_{j}\right) \in \bar{S}$ then (provided $C_{3}$ has been chosen large enough) Lemma 4.2 implies there are three subsets $F_{1}, F_{2}, F_{3}$ of the set $A_{t, \epsilon}\left(x_{j}, r_{j}\right)$ such that $\operatorname{dist}\left(F_{l}, F_{m}\right) \geq 2 C_{3}^{-1} \bar{\lambda}, l \neq m$, and $\left|F_{l}\right| \gtrsim \delta \bar{\lambda}$ for each $l$. For fixed $l$, we let $S_{l}$ be those indices $i \in S_{t, \epsilon}\left(x_{j}, r_{j}\right)$ such that $F_{l} \cap C_{\delta}\left(x_{i}, r_{i}\right) \neq \emptyset$. The sets $C_{\delta}\left(x_{i}, r_{i}\right), i \in S_{l}$ must cover $F_{l}$ at least $\bar{\mu}$ times. So

$$
\sum_{i \in S_{l}}\left|F_{l} \cap C_{\delta}\left(x_{i}, r_{i}\right)\right| \gtrsim \bar{\mu} \bar{\lambda} \delta
$$

Also, for each fixed $i$ we have $\left|F_{l} \cap C_{\delta}\left(x_{i}, r_{i}\right)\right| \lesssim \frac{\delta^{2}}{\sqrt{t \epsilon}}$ by (16). Consequently

$$
\operatorname{card} S_{l} \gtrsim \delta^{-1} \bar{\mu} \bar{\lambda} \sqrt{t \epsilon} \text {. }
$$

Estimate (17) implies that if $i \in S_{t, \epsilon}\left(x_{j}, r_{j}\right)$ then the diameter of $C_{\delta}\left(x_{i}, r_{i}\right) \cap C_{\delta}\left(x_{j}, r_{j}\right)$ is $\lesssim \sqrt{\frac{\epsilon}{t}}$, which is small compared with $\bar{\lambda}$ if $C_{2}$ has been chosen large enough. So if $l \neq m, i \in S_{l}, k \in S_{m}$, then the distance between $C_{\delta}\left(x_{i}, r_{i}\right) \cap C_{\delta}\left(x_{j}, r_{j}\right)$ and $C_{\delta}\left(x_{k}, r_{k}\right) \cap C_{\delta}\left(x_{j}, r_{j}\right)$ is $\geq C_{3}^{-1} \bar{\lambda}$. We conclude that if $i_{l} \in S_{l}$ for $l=1,2,3$ then $\left(j, i_{1}, i_{2}, i_{3}\right) \in Q$. So

$$
\operatorname{card} Q \gtrsim \bar{M}\left(\delta^{-1} \bar{\mu} \bar{\lambda} \sqrt{t \epsilon}\right)^{3} .
$$

If we compare this equation with (57) we obtain

$$
\bar{\mu}^{3} \lesssim \frac{\delta^{2}}{t^{\frac{3}{2}} \epsilon^{\frac{1}{2}}} \bar{\lambda}^{-5} \min \left(M, \frac{t}{\delta}\right)^{2} \frac{M}{\bar{M}},
$$

or equivalently

$$
\mu^{3} \lesssim a(t, \epsilon)^{-9} \frac{\delta^{2}}{t^{\frac{3}{2}} \epsilon^{\frac{1}{2}}} \lambda^{-5} \min \left(M, \frac{t}{\delta}\right)^{2}
$$

\footnotetext{
${ }^{5}$ The hypotheses on $\epsilon, t$ and $\lambda$ in Lemma 3.1(ii) are satisfied provided $C_{2}$ is large enough.
} 
This can be rewritten as

$$
\mu^{3} \lesssim M^{\frac{1}{2}} \lambda^{-5} \cdot\left\{\begin{array}{lr}
a(t, \epsilon)^{-9}\left(\frac{\delta}{\epsilon}\right)^{\frac{1}{2}}\left(\frac{t}{\delta M}\right)^{\frac{1}{2}} & \text { if } M \geq \frac{t}{\delta} \\
a(t, \epsilon)^{-9}\left(\frac{\delta}{\epsilon}\right)^{\frac{1}{2}}\left(\frac{M \delta}{t}\right)^{\frac{3}{2}} & \text { if } M \leq \frac{t}{\delta}
\end{array}\right.
$$

The expression inside the brace is bounded by a constant by the definition of $a(t, \epsilon)$, provided the constant $\alpha$ is less than $\frac{1}{18}$. So we have proved (53) in case (i).

In case (ii), we fix $j$ with $C\left(x_{j}, r_{j}\right) \in \bar{S}$ and make the trivial estimate card $S_{t \epsilon}\left(x_{j}, r_{j}\right) \lesssim \min \left(M, \frac{t}{\delta}\right)$. It follows that

$$
\begin{aligned}
\bar{\mu} \bar{\lambda} \delta & \lesssim \sum_{i \in S_{t} \epsilon}\left(x_{j}, r_{j}\right) \\
& \lesssim \min \left(M, \frac{t}{\delta}\right) \frac{\delta^{2}}{\sqrt{t \epsilon}},
\end{aligned}
$$

where we used (16). Thus $\bar{\mu} \lesssim \bar{\lambda}^{-1} \sqrt{\frac{t}{\epsilon}} \min \left(\frac{M \delta}{t}, 1\right)$. Using the hypothesis (ii) we therefore have

$$
\bar{\mu} \lesssim \bar{\lambda}^{-\frac{5}{3}}\left(\frac{t}{\epsilon}\right)^{\frac{1}{6}} \min \left(\frac{M \delta}{t}, 1\right)
$$

i.e.,

$$
\begin{aligned}
\mu & \lesssim a(t, \epsilon)^{-\frac{8}{3}} \lambda^{-\frac{5}{3}}\left(\frac{t}{\epsilon}\right)^{\frac{1}{6}} \min \left(\frac{M \delta}{t}, 1\right) \\
& =\lambda^{-\frac{5}{3}} M^{\frac{1}{6}} \cdot \begin{cases}a(t, \epsilon)^{-\frac{8}{3}}\left(\frac{\delta}{\epsilon}\right)^{\frac{1}{6}}\left(\frac{t}{\delta M}\right)^{\frac{1}{6}} & \text { if } M \geq \frac{t}{\delta} \\
a(t, \epsilon)^{-\frac{8}{3}}\left(\frac{\delta}{\epsilon}\right)^{\frac{1}{6}}\left(\frac{M \delta}{t}\right)^{\frac{5}{6}} & \text { if } M \leq \frac{t}{\delta}\end{cases}
\end{aligned}
$$

The expression in the brace is bounded by a constant provided $\alpha$ has been chosen less than $\frac{1}{16}$, so we have proved (53).

Completion of proof of Theorem 4.1. With notation as above we have

$$
\begin{aligned}
|E| & \geq\left|\left\{x \in E: \operatorname{card}\left(\left\{i: x \in C_{\delta}\left(x_{i}, r_{i}\right) \leq \mu\right\}\right)\right\}\right| \\
& \geq \mu^{-1} \sum_{j}\left|\left\{x \in E \cap C_{\delta}\left(x_{j}, r_{j}\right): \operatorname{card}\left(\left\{i: x \in C_{\delta}\left(x_{i}, r_{i}\right)\right\}\right) \leq \mu\right\}\right| \\
& \gtrsim \mu^{-1} M \lambda \delta \\
& \gtrsim \lambda^{\frac{8}{3}} M^{\frac{5}{6}} \delta
\end{aligned}
$$

by (53). Consequently $(M \delta)^{\frac{5}{6}} \lesssim \frac{|E|}{\delta^{\frac{1}{6}} \lambda^{\frac{8}{3}}}$ and Theorem 4.1 is proved. 
Proof of Theorem 2'. It suffices to prove Theorem $2^{\prime}$ for functions $f$ with support in the unit disc. Therefore, we need only observe (as is done for example in $[\mathbf{1 0}]$ ) that the function

$$
\Phi(x, y)=|x-y|
$$

satisfies the conditions (14), (15) at all points where $x \neq y$, and then use a partition of unity to reduce to Theorem 4.1.

Remark. One can consider various other cases besides Theorem $2^{\prime}$ and Lemma 5.1 below. For example, one can consider circles with one of the coordinates of the center taken as the parameter $r$, instead of the radius. One gets the following result: If a set in $\mathbb{R}^{2}$ contains circles with centers at all points of a smooth curve, then the Hausdorff dimension of $E$ is at least $\frac{11}{6}$, and the estimate (49) holds for the restriction of the circular maximal function to the curve. The proof is done most easily by using the remark at the beginning of Section 5 below. We note that $E$ can have measure zero; cf. [12] (this reference was pointed out by W. Schlag).

\section{Proof of Theorem $3^{\prime}$.}

It is convenient to restate Theorem 4.1 using a different way of presenting the curve family (cf. also [10] in this connection). Suppose then that $U \subset$ $\mathbb{R}^{2} \times \mathbb{R}^{3}, \Psi: U \rightarrow \mathbb{R}$. We will denote variables in $\mathbb{R}^{2} \times \mathbb{R}^{3}$ by $(x, \lambda), x \in$ $\mathbb{R}^{2}, \lambda \in \mathbb{R}^{3}$. Define $C(\lambda)=\left\{x \in \mathbb{R}^{2}: \Psi(x, \lambda)=0\right\}$ and let $C_{\delta}(\lambda)$ be its $\delta$ neighborhood. Fix a point $\left(a, \lambda_{0}\right) \in U$, and let $r_{0}$ be the third coordinate of $\lambda_{0}$. Assume the following: $\Psi\left(a, \lambda_{0}\right)=0, \nabla_{x} \Psi\left(a, \lambda_{0}\right) \neq 0, \nabla_{\lambda} \Psi\left(a, \lambda_{0}\right) \neq 0$, and the cinematic curvature condition, i.e., that if $x$ is close to $a$ and $\lambda, \mu$ are close to $\lambda_{0}$, and $C(\lambda)$ and $C(\mu)$ intersect at $x$, then either the unit tangent vectors to $C(\lambda)$ and $C(\mu)$ at $x$, or else the curvatures of $C(\lambda)$ and $C(\mu)$ at $x$, differ by $\gtrsim|\lambda-\mu|$. If $f$ is supported in a small neighborhood of $a, \delta$ is small, $z \in\left(r_{0}-\epsilon_{0}, r_{0}+\epsilon_{0}\right)$ then define a maximal function $M_{\delta} f(z)$ via

$$
M_{\delta} f(z)=\sup _{\lambda} \delta^{-1} \int_{C_{\delta}(\lambda)}|f|,
$$

where the sup is taken over all parameter values $\lambda$ with $\left|\lambda-\lambda_{0}\right|<\epsilon_{0}$ and $\lambda_{(3)}=z$. Then the estimate (49) is valid.

The proof is as follows: The maximal function is unaffected by the change of variables $\lambda \rightarrow\left(\lambda_{(1)}, \lambda_{(2)}+\lambda_{(3)}, \lambda_{(3)}\right)$ or $\lambda \rightarrow\left(\lambda_{(1)}+\lambda_{(3)}, \lambda_{(2)}, \lambda_{(3)}\right)$, so we may assume that $\frac{\partial \Psi}{\partial \lambda_{(3)}}\left(a, \lambda_{0}\right) \neq 0$. But then by the implicit function theorem the equation $\Psi(x, \lambda)=0$ can be solved for $\lambda_{3}$ and we are reduced to the situation of Theorem 4.1. 
We will now estimate the maximal function defined by (7) by applying this form of Theorem 4.1 with

$$
\Psi((s, t),(x, y, z))=s^{2}-\frac{(t-y)^{2}}{z}-x .
$$

Lemma 5.1. If $p<\frac{8}{3}$ and $q=2 p^{\prime}$, and if $f: \mathbb{R}^{2} \rightarrow \mathbb{R}$ satisfies $\operatorname{supp} f \subset$ $\left\{(s, t): \frac{1}{2} \leq s \leq 2\right\}$, then

$$
\left\|\tilde{M}_{\delta} f\right\|_{L^{q}\left(\left\{z: \frac{1}{2}<z<2\right\}\right)} \lesssim \delta^{-\frac{1}{2}\left(\frac{3}{p}-1\right)}\|f\|_{p} .
$$

Proof. One can easily check the cinematic curvature condition for the family of curves $H(x, y, z)$ in the region $\frac{1}{2} \leq s \leq 2$, by representing the curves as graphs over the $t$ axis and using the formulas from first year calculus. We omit this calculation. Using Theorem 4.1 and the above remarks it follows that Lemma 5.1 is valid locally, i.e., is valid with the additional assumption that $\operatorname{supp} f \subset\{(s, t):-1 \leq t \leq 1\}$. The definition of $\tilde{M}_{\delta}$ is translation invariant in $t$, so the lemma is valid with bounds uniform in $N$ provided that $\operatorname{supp} f \subset Q_{N}$ for some $N$, where $Q_{N}=\left[\frac{1}{2}, 2\right] \times[N-1, N+1]$. On the other hand, when $z \in\left[\frac{1}{2}, 2\right]$ it is easy to check that $H_{\delta}(x, y, z)$ intersects only a bounded number of $Q_{N}$ 's. Hence $\tilde{M}_{\delta} f \lesssim \sup _{N} \tilde{M}_{\delta}\left(\chi_{Q_{N}} f\right)$ and then the lemma follows in a standard way, namely:

$$
\begin{aligned}
\left\|\tilde{M}_{\delta} f\right\|_{q} & \leq\left\|\left(\sum_{N} \tilde{M}_{\delta}\left(\chi_{Q_{N}} f\right)^{q}\right)^{\frac{1}{q}}\right\|_{q} \\
& =\left(\sum_{N}\left\|\tilde{M}_{\delta}\left(\chi_{Q_{N}} f\right)\right\|_{q}^{q}\right)^{\frac{1}{q}} \\
& \lesssim \delta^{-\frac{1}{2}\left(\frac{3}{p}-1\right)}\left(\sum\left\|\chi_{Q_{N}} f\right\|_{p}^{q}\right)^{\frac{1}{q}} \\
& \lesssim \delta^{-\frac{1}{2}\left(\frac{3}{p}-1\right)}\|f\|_{p},
\end{aligned}
$$

since the $Q_{N}$ have finite overlap and $q \geq p$.

We will relate the Kakeya maximal function of a rotation invariant function to the maximal function $\tilde{M}_{\delta}$. In order to do so we first recall the standard definitions of the x-ray transform $R F$ and maximal x-ray transform $R^{*} F$, for functions $F: \mathbb{R}^{3} \rightarrow \mathbb{R}$. Namely

$$
R F(\ell)=\int_{\ell} F d \sigma
$$

Here $\ell$ denotes a line in $\mathbb{R}^{3}$ and $d \sigma$ is arc length. Also, $R^{*} F: \mathbb{P}^{2} \rightarrow \mathbb{R}$,

$$
R^{*} F(e)=\sup _{\ell: e_{\ell}=e} R F(\ell)
$$


where $e_{\ell}$ is the direction of the line $\ell$.

Similarly, if $f$ is a function on $\mathbb{R}^{2}$ then we define $\mathcal{H} f:\left\{(x, y, z) \in \mathbb{R}^{3}\right.$ : $\left.x>0, \frac{1}{2} \leq z \leq 2\right\} \rightarrow \mathbb{R}$ via

$$
\mathcal{H} f(x, y, z)=\int_{H(x, y, z)} f(s, t) d t
$$

and $\mathcal{H}^{*} f:\left\{z \in \mathbb{R}: \frac{1}{2} \leq z \leq 2\right\} \rightarrow \mathbb{R}$ via

$$
\mathcal{H}^{*} f(z)=\sup _{\substack{x>0 \\ y \in \mathbb{R}}} \mathcal{H} f(x, y, z) .
$$

We will use complex notation for the first two variables in $\mathbb{R}^{3}$ when convenient, i.e., will denote the point $\left(x_{1}, x_{2}, x_{3}\right)$ by $\left(x_{1}+i x_{2}, x_{3}\right)$. If $\ell$ is a line in $\mathbb{R}^{3}$ which is not parallel to the $x_{3}$ axis then $\ell$ can be parametrized by its direction $\left(e^{i \theta}, \sqrt{z}\right) \in \mathbb{P}^{2}, z \geq 0$, and its closest point to the $e_{3}$ axis, which will be of the form $\left(i \alpha e^{i \theta}, y\right), \alpha \in \mathbb{R}, y \in \mathbb{R}$. In this notation we have:

Lemma 5.2. If $F$ is a rotation invariant function in $\mathbb{R}^{3}, F(x)=$ $f\left(\sqrt{x_{1}^{2}+x_{2}^{2}}, x_{3}\right)$, then for any line $\ell$ which is not parallel to the $x_{3}$ axis and which satisfies $z \neq 0$,

$$
R F(\ell)=\sqrt{\frac{1+z}{z}} \mathcal{H} f\left(\alpha^{2}, y, z\right) .
$$

Proof. We have

$$
R F(\ell)=\int F\left(\left(i \alpha e^{i \theta}, y\right)+\tau\left(e^{i \theta}, \sqrt{z}\right)\right) \sqrt{1+z} d \tau,
$$

and since $F$ is rotation invariant this implies

$$
\begin{aligned}
R F(\ell) & =\int_{0}^{2 \pi} \int F\left(e^{i \phi}\left(i \alpha e^{i \theta}+\tau e^{i \theta}\right), y+\tau \sqrt{z}\right) \sqrt{1+z} d \tau \frac{d \phi}{2 \pi} \\
& =\int_{0}^{2 \pi} F\left((\tau+i \alpha) e^{i(\theta+\phi)}, y+\tau \sqrt{z}\right) \sqrt{1+z} d \tau \frac{d \phi}{2 \pi} \\
& =\int_{0}^{2 \pi} F\left(\left(\frac{t-y}{\sqrt{z}}+i \alpha\right) e^{i(\theta+\phi)}, t\right) \sqrt{\frac{1+z}{z}} d t \frac{d \phi}{2 \pi} \\
& =\int f\left(\left|\frac{t-y}{\sqrt{z}}+i \alpha\right|, t\right) d t \sqrt{\frac{1+z}{z}} \\
& =\int_{H\left(\alpha^{2}, y, z\right)} f(s, t) d t \sqrt{\frac{1+z}{z}}
\end{aligned}
$$

as claimed. 
We define $\Omega_{\rho} \subset \mathbb{P}^{2}$ via $\Omega_{\rho}=\left\{e: \sqrt{z} \in\left[\frac{\rho}{\sqrt{2}}, \sqrt{2} \rho\right]\right\}$, and $\tilde{\Omega}=\left\{e \in \mathbb{P}^{2}\right.$ : $\left.\sqrt{z} \in\left[\delta, \frac{1}{\delta}\right]\right\}$. We also define a variant of $F_{\delta}^{*}$, as follows:

$$
\bar{F}_{\delta}(e) \stackrel{\text { def }}{=} \delta^{-2} \sup _{t} \int_{t}|F|,
$$

where $t$ runs over $\delta$-neighborhoods of lines $\ell$ with $e_{\ell}=e$. Thus $\bar{F}_{\delta}(e) \gtrsim$ $F_{\delta}^{*}(e)$, and $\bar{F}_{\delta}(e) \approx F_{\delta}^{*}(e)$ if $z \leq 1$ and $f$ is supported in $\{(s, t): s \leq 2\}$.

Remark. At this point we are in a position to prove the Hausdorff dimension statement, Theorem 3. We only sketch the argument since of course Theorem 3 is also a corollary of Theorem $3^{\prime}$. Suppose $F$ and $f$ are as in Lemma 5.2, $F \geq 0$. By applying Lemma 5.2 to the convolution of $F$ with the characteristic function of a disc of radius $\delta$, we obtain

$$
F_{\delta}^{*}(e) \lesssim \tilde{M}_{\delta} f(z) \forall e \in \Omega_{1} .
$$

Consequently by Lemma 5.1,

$$
\left\|F_{\delta}^{*}\right\|_{L^{q}\left(\Omega_{1}\right)} \lesssim \delta^{-\frac{1}{2}\left(\frac{3}{p}-1\right)}\|F\|_{p}, \quad p<\frac{8}{3}, q=2 p^{\prime}
$$

for rotation invariant functions supported in $\left\{x \in \mathbb{R}^{3}: \sqrt{x_{1}^{2}+x_{2}^{2}} \in\left[\frac{1}{2}, 2\right]\right\}$. It is clear that the interval $\left[\frac{1}{2}, 2\right]$ here could be replaced by any other closed bounded interval not containing the origin. If $E$ is a rotation invariant set which contains a line segment in every direction, then its intersection with $\sqrt{x_{1}^{2}+x_{2}^{2}} \in\left[a, \frac{1}{a}\right]$ contains a segment in a positive measure set of $\Omega_{1}$ directions for suitable $a>0$. We may then obtain the dimension statement by applying (60) to the characteristic function of the latter set.

The purpose of the contortions below is to pass from estimates like (60) to the scale invariant $L^{p} \rightarrow L^{q}$ estimates of Theorem $3^{\prime}$. As motivation, we note that (by the preceding remark) Theorem $3^{\prime}$ and (60) have essentially the same geometric content, and on the other hand the values of $p$ in Theorem $3^{\prime}$ are larger than those in (60). It is therefore natural to interpolate between an estimate like (60) and an $L^{\infty} \rightarrow L^{\infty}$ estimate. This is in fact what we will do; cf. (77) below.

Lemma 5.3. Assume that $p<\frac{17}{6}$ and that $p$ is sufficiently close to $\frac{17}{6} .{ }^{6}$ Let $q=2 p^{\prime}$. Then there is $\epsilon=\epsilon_{p}>0$ such that if $F$ and $f$ are as in Lemma 5.2 and $\operatorname{supp} f \subset\left\{(s, t): \frac{1}{2} \leq s \leq 2\right\}$ then

$$
\left\|\bar{F}_{\delta}\right\|_{L^{q}(\tilde{\Omega})} \lesssim \delta^{-\left(\frac{3}{p}-1\right)+\epsilon}\|F\|_{p}
$$

Proof. We always assume $F \geq 0$. It suffices to prove

$$
\left\|\bar{F}_{\delta}\right\|_{L^{q}\left(\Omega_{\rho}\right)} \lesssim \delta^{-\left(\frac{3}{p}-1\right)+\epsilon}\|F\|_{p}
$$

\footnotetext{
6 "sufficiently close to $\frac{17}{6}$ " can be taken to mean that $p>\frac{5}{3}$.
} 
with $\epsilon>0$ independent of $\rho$, since then we obtain the estimate of the lemma with a slightly smaller value of $\epsilon$ by summing over dyadic $\rho$ from $\delta$ to $\frac{1}{\delta}$. To prove (61) we consider two cases, (i) $\rho \leq 1$ and (ii) $\rho \geq 1$.

In case (i) we fix $\rho$ and define a map $T: \mathbb{R}^{3} \rightarrow \mathbb{R}^{3}$ via $T\left(x_{1}, x_{2}, x_{3}\right)=$ $\left(x_{1}, x_{2}, \rho x_{3}\right)$ Then the action of $T$ on $\mathbb{P}^{2}$ is given by

$$
\left(e^{i \theta}, \sqrt{z}\right)=e \Rightarrow e_{T} \stackrel{\text { def }}{=}\left(e^{i \theta}, \rho \sqrt{z}\right)
$$

i.e.,

$$
(1+z)^{-\frac{1}{2}} R^{*}(F \circ T)(e)=\left(1+\rho^{2} z\right)^{-\frac{1}{2}} R^{*} F\left(e_{T}\right) .
$$

Note that $e \in \Omega_{1} \leftrightarrow e_{T} \in \Omega_{\rho}$. Also let $\Phi_{\delta \sigma}$ be the characteristic function of the set $\left\{x \in \mathbb{R}^{3}: \sqrt{x_{1}^{2}+x_{2}^{2}}<\delta,\left|x_{3}\right|<\sigma\right\}$, normalized to have $L^{1}$ norm 1 , and similarly let $\phi_{\delta \sigma}$ be the $L^{1}$ normalized characteristic function of the set $\left\{(s, t) \in \mathbb{R}^{2}:|s|<2 \delta,|t|<\sigma\right\}$. If $G=F \circ T$ and $g$ is defined via $G(x)=g\left(\sqrt{x_{1}^{2}+x_{2}^{2}}, x_{3}\right)$, and if $e \in \Omega_{1}$, then standard arguments together with (62) and Lemma 5.2 justify the following string of inequalities:

$$
\begin{aligned}
F_{\delta}^{*}\left(e_{T}\right) & \lesssim R^{*}\left(F * \Phi_{\delta \delta}\right)\left(e_{T}\right) \\
& \approx R^{*}\left(\left(F * \Phi_{\delta \delta}\right) \circ T\right)(e) \\
& =R^{*}\left(G * \Phi_{\delta \frac{\delta}{\rho}}\right)(e) \\
& \lesssim \mathcal{H}^{*}\left(g * \phi_{\delta \frac{\delta}{\rho}}\right)(z) .
\end{aligned}
$$

Taking into account that the map $e \rightarrow e_{T}$ distorts areas by a factor of roughly $\rho$ we therefore have

$$
\left\|F_{\delta}^{*}\right\|_{L^{q}\left(\Omega_{\rho}\right)} \lesssim \rho^{\frac{1}{q}}\left\|\mathcal{H}^{*}\left(g * \phi_{\delta} \frac{\delta}{\rho}\right)\right\|_{L^{q}\left(\left\{z: \frac{1}{2}<z<2\right\}\right)},
$$

and it remains to estimate the latter expression.

Claim. Let $\sigma \leq 1, \delta \leq C_{0} \sigma$, and assume also that $\delta<\frac{1}{10}$. Fix $x>0, y \in$ $\mathbb{R}, z \in\left[\frac{1}{2}, 2\right]$, let $H=H(x, y, z)$ and let $\mu_{H}$ be the measure defined via $\int f d \mu_{H}=\int_{H(x, y, z)} f(s, t) d t$. Let $H_{\alpha}=H_{\alpha}(x, y, z), S(y, \beta)=\left\{(s, t) \in \mathbb{R}^{2}\right.$ : $\left.\frac{1}{2} \leq s \leq 2,|t-y| \leq C_{1} \beta\right\}$ for an appropriate constant $C_{1}$. Then when $\frac{1}{2} \leq s \leq t$,

$$
\phi_{\delta \sigma} * \mu_{H}(s, t) \lesssim \sum_{\alpha} \alpha^{-1} \chi_{H_{\alpha} \cap S\left(y, \frac{\alpha}{\sigma}\right)}(s, t)+R .
$$

In the sum, $\alpha$ runs over all numbers of the form $2^{j} \max \left(\sigma^{2}, \delta\right), j \in \mathbb{Z}^{+} \cup$ $\{0\}, \alpha \leq \sigma$, and $R$ satisfies

$$
R=0, \text { if } \sigma^{2} \leq \delta
$$

$$
\operatorname{supp} R \subset\left\{(s, t):|t-y| \leq C \sigma,|s-\sqrt{x}| \leq C \sigma^{2}\right\} \quad \text { and }
$$




$$
|R(s, t)| \lesssim(\delta \sigma)^{-1} \min \left(\sqrt{\delta}, \frac{\delta}{\sqrt{|s-\sqrt{x}|}}\right), \quad \text { if } \sigma^{2}>\delta .
$$

Proof of the claim. This is a standard type of estimation using (implicitly) the curvature of the curves $H(x, y, z)$.

Fix a point $\left(s_{0}, t_{0}\right)$ with $\frac{1}{2} \leq s_{0} \leq 2$; we will prove the claim at the point $\left(s_{0}, t_{0}\right)$. Let $B$ be the rectangle centered at $\left(s_{0}, t_{0}\right)$ with sides parallel to the axes, of lengths $4 \delta$ in t he $s$ direction and $2 \sigma$ in the $t$ direction. We always assume that $B \cap H \neq \emptyset$ which is evidently satisfied if $\phi_{\delta \sigma} * \mu_{H}\left(s_{0}, t_{0}\right) \neq 0$. We will also use the notation $(s(t), t)$ to denote points of $H$; thus

$$
s(t)=\sqrt{x}+\frac{(t-y)^{2}}{z} .
$$

We split the proof of the claim into three cases.

(i) $\left|t_{0}-y\right| \geq 2 \sigma$.

Then $\left|t-t_{0}\right| \leq \sigma$ implies (cf. (66)) that

$$
\left|\frac{\partial s(t)}{\partial t}\right| \approx\left|t_{0}-y\right| \text {. }
$$

Formula (67) immediately implies that $\mu_{H}(B \cap H) \lesssim \min \left(\frac{\delta}{\left|t_{0}-y\right|}, \sigma\right)$, i.e.,

$$
\left|\phi_{\delta \sigma} * \mu_{H}\right| \lesssim(\delta \sigma)^{-1} \min \left(\frac{\delta}{\left|t_{0}-y\right|}, \sigma\right) \approx\left(\delta+\sigma\left|t_{0}-y\right|\right)^{-1} .
$$

Next, since $B \cap H$ is nonempty we can fix $t$ with $\left|t-t_{0}\right| \leq \sigma$ and $\left|s(t)-s_{0}\right| \leq$ $2 \delta$. Then $\left|s_{0}-s\left(t_{0}\right)\right| \leq\left|s_{0}-s(t)\right|+\left|s(t)-s\left(t_{0}\right)\right| \lesssim \delta+\sigma\left|t_{0}-y\right|$, where we used (67). Thus $\left(s_{0}, t_{0}\right) \in H_{\alpha} \cap S\left(y, \frac{\alpha}{\sigma}\right)$, with $\alpha \approx \delta+\sigma\left|t_{0}-y\right|$. This and (68) imply the claim (with $R=0$ ) in case (i).

(ii) $\left|t_{0}-y\right| \leq 2 \sigma$, and $\sigma^{2} \leq \delta$.

In this case we just use that $\mu_{H}(B \cap H) \leq \sigma$, i.e.,

$$
\left|\phi_{\delta \sigma} * \mu_{H}\left(s_{0}, t_{0}\right)\right| \lesssim \delta^{-1} \approx\left(\delta+\sigma^{2}\right)^{-1}
$$

Also (by (66))

$$
\left|\frac{\partial s(t)}{\partial t}\right| \lesssim \sigma
$$

when $\left|t-t_{0}\right| \leq \sigma$. Fix $t$ with $\left|t-t_{0}\right| \leq \sigma$ and $\left|s(t)-s_{0}\right| \leq 2 \delta$. Then

$$
\left|s_{0}-s\left(t_{0}\right)\right| \leq\left|s_{0}-s(t)\right|+\left|s(t)-s\left(t_{0}\right)\right| \lesssim \delta+\sigma^{2} .
$$

We used (70). It follows that $\left(s_{0}, t_{0}\right) \in H_{\alpha} \cap S\left(y, \frac{\alpha}{\sigma}\right), \alpha \approx \delta+\sigma^{2}$. The claim (with $R=0$ ) follows from this and (69).

(iii) $\left|t_{0}-y\right| \leq 2 \sigma$, and $\sigma^{2} \geq \delta$. 
In this case we set $R\left(s_{0}, t_{0}\right)=\phi_{\delta \sigma} * \mu_{H}$. It suffices to show that (65) then holds. From (66) we easily obtain the following two estimates:

$$
\begin{aligned}
\left|\frac{\partial s(t)}{\partial t}\right| & \approx \sqrt{s(t)-\sqrt{x}} \\
\left|\frac{\partial^{2} s(t)}{\partial t^{2}}\right| & \approx 1
\end{aligned}
$$

when $\left|t-t_{0}\right|$ is small. The first estimate implies that if $\left|s_{0}-\sqrt{x}\right|$ is large compared with $\delta$, then $\mu_{H}(B \cap H) \lesssim \frac{\delta}{\sqrt{\left|s_{0}-\sqrt{x}\right|}}$. The second estimate implies that in all cases $\mu_{H}(B \cap H) \lesssim \sqrt{\delta}$. Combining these, we get $\mu_{H}(B \cap H) \lesssim$ $\min \left(\sqrt{\delta}, \frac{\delta}{\sqrt{\left|s_{0}-\sqrt{x}\right|}}\right)$ in all cases, which gives the estimate for $|R(s, t)|$ in (65). On the other hand, we're assuming $\left|t_{0}-y\right| \leq 2 \sigma$, and the proof of (71) did not use that $\sigma^{2} \leq \delta$, so (71) is valid in the present case also. Using (70) once more it follows that $\left|s\left(t_{0}\right)-\sqrt{x}\right| \lesssim \sigma^{2}$, so $\left|s_{0}-\sqrt{x}\right| \lesssim \sigma^{2}$ and the claim is completely proved.

Applying the claim with $\sigma=\frac{\delta}{\rho}$, we estimate the right hand side of (63) as follows. For each $z \in\left[\frac{1}{2}, 2\right]$,

$$
\mathcal{H}^{*}\left(g * \phi_{\delta} \frac{\delta}{\rho}\right)(z) \lesssim \sum_{\alpha} \sup _{y} \tilde{M}_{\alpha}\left(\chi_{S\left(y, \frac{\alpha \rho}{\delta}\right)} g\right)(z)+E(z) .
$$

Here $\alpha$ runs over all numbers of the form $2^{j} \max \left(\delta, \frac{\delta^{2}}{\rho^{2}}\right)$ with $j \geq 0$ and $\alpha \leq \frac{\delta}{\rho}$, and $E(z) \leq\|g R\|_{1}$ where $R$ is a function (depending on $z$ ) which satisfies the estimates (65) for a certain $s_{0}$ and $t_{0}$, with $\sigma=\frac{\delta}{\rho}$. We first discuss the last term in (72). Estimates (65) imply that if $\delta>\rho^{2}$ then

$$
\begin{aligned}
\|R\|_{p^{\prime}}^{p^{\prime}} & \lesssim\left(\frac{\rho}{\delta}\right)^{p^{\prime}} \int_{|t-y| \leq \frac{\delta}{\rho}} \int_{|s-\sqrt{x}| \leq \frac{\delta^{2}}{\rho^{2}}}|s-\sqrt{x}|^{-\frac{p^{\prime}}{2}} d s d t \\
& \approx\left(\frac{\rho}{\delta}\right)^{2 p^{\prime}-3}
\end{aligned}
$$

i.e., $\|R\|_{p^{\prime}} \lesssim\left(\frac{\rho}{\delta}\right)^{\frac{3}{p}-1}$, and of course $R=0$ if $\delta \leq \rho^{2}$. Using this together with Hölder's inequality and the change of variables formula $\|g\|_{p}=\rho^{-\frac{1}{p}}\|f\|_{p}$, we obtain

$$
\begin{aligned}
\rho^{\frac{1}{q}}\|E\|_{q} \leq \rho^{\frac{1}{q}}\|E\|_{\infty} & \lesssim \begin{cases}\left(\frac{\rho}{\delta}\right)^{\frac{3}{p}-1} \rho^{\frac{1}{q}-\frac{1}{p}}\|f\|_{p} & \text { if } \rho \leq \delta^{\frac{1}{2}} \\
0 & \text { if } \rho \geq \delta^{\frac{1}{2}}\end{cases} \\
& \lesssim \delta^{-\frac{3}{4}\left(\frac{3}{p}-1\right)}\|f\|_{p} .
\end{aligned}
$$


The exponent $-\frac{3}{4}\left(\frac{3}{p}-1\right)$ here is of course of the form $-\left(\frac{3}{p}-1\right)+\epsilon$, so, substituting (72) into (63), we see that it suffices to prove

$$
\rho^{\frac{1}{q}} \sum_{\alpha}\left\|\sup _{y} \tilde{M}_{\alpha}\left(g \chi_{S\left(y, \frac{\alpha \rho}{\delta}\right)}\right)\right\|_{q} \lesssim \delta^{-\left(\frac{3}{p}-1\right)+\epsilon}\|f\|_{p} .
$$

In order to prove (73) we fix $\alpha$ and let $\left\{I_{j}\right\}$ be a covering of $\mathbb{R}$ by intervals of length $3 C \frac{\alpha \rho}{\delta}$, with finite overlap. Here $C$ is the constant in the definition of $S(y, \beta)$. Then we let $S_{j}=\left[\frac{1}{2}, 2\right] \times I_{j} \subset \mathbb{R}^{2}$. We have

$$
\begin{aligned}
\rho^{\frac{1}{q}}\left\|\sup _{y} \tilde{M}_{\alpha}\left(g \chi_{S\left(y, \frac{\alpha \rho}{\delta}\right)}\right)\right\|_{q} & \lesssim \rho^{\frac{1}{q}}\left\|\sup _{j} \tilde{M}_{\alpha}\left(g \chi_{S_{j}}\right)\right\|_{q} \\
& \lesssim \rho^{\frac{1}{q}}\left\|\left(\sum_{j} \tilde{M}_{\alpha}\left(g \chi_{S_{j}}\right)^{q}\right)^{\frac{1}{q}}\right\| \|_{q} \\
& =\rho^{\frac{1}{q}}\left(\sum_{j}\left\|\tilde{M}_{\alpha}\left(g \chi_{S_{j}}\right)\right\|_{q}^{q}\right)^{\frac{1}{q}}
\end{aligned}
$$

with all $L^{q}$ norms being taken over $\frac{1}{2}<z<2$, and we now proceed to bound the terms in the sum over $j$. We have an $L^{\infty} \rightarrow L^{\infty}$ estimate

$$
\left\|\tilde{M}_{\alpha} h\right\|_{\infty} \lesssim \frac{\alpha \rho}{\delta}\|h\|_{\infty} \quad \text { if } \operatorname{supp} h \subset S_{j},
$$

since each set $H_{\alpha}(x, y, z)$ intersects $S_{j}$ in measure $\lesssim \alpha \cdot \frac{\alpha \rho}{\delta}$. Now fix $p_{0}$ with

$$
2 p-3<p_{0}<\frac{8}{3} \text {. }
$$

This is possible since $p<\frac{17}{6}$. Let $q_{0}=2 p_{0}^{\prime}$. Interpolating the bound (75) with the bound

$$
\left\|\tilde{M}_{\alpha} h\right\|_{q_{0}} \lesssim \alpha^{-\frac{1}{2}\left(\frac{3}{p_{0}}-1\right)}\|h\|_{p_{0}}
$$

of Lemma 5.1, we obtain

$$
\left\|\tilde{M}_{\alpha} h\right\|_{\bar{q}} \lesssim \alpha^{-\frac{1}{2}\left(\frac{3}{p_{0}}-1\right) \frac{p_{0}}{p}}\left(\frac{\alpha \rho}{\delta}\right)^{1-\frac{p_{0}}{p}}\|h\|_{p} \text { if supp } h \subset S_{j} .
$$

In (77), the number $\bar{q}$ is equal to $q_{0} \frac{p}{p_{0}}$ and therefore larger than $q$. It follows that (77) remains valid with $\bar{q}$ replaced by $q$. Applying (77) with this modification to the terms in the sum (74), we obtain

$$
(74) \lesssim \rho^{\frac{1}{q}} \alpha^{-\frac{1}{2}\left(\frac{3}{p_{0}}-1\right) \frac{p_{0}}{p}}\left(\frac{\alpha \rho}{\delta}\right)^{1-\frac{p_{0}}{p}}\left(\sum_{j}\left\|g \chi_{S_{j}}\right\|_{p}^{q}\right)^{\frac{1}{q}}
$$




$$
\lesssim \rho^{\frac{1}{q}} \alpha^{-\frac{1}{2}\left(\frac{3}{p_{0}}-1\right) \frac{p_{0}}{p}}\left(\frac{\alpha \rho}{\delta}\right)^{1-\frac{p_{0}}{p}}\|g\|_{p}
$$

The last line follows since $q>p$ and the sets $S_{j}$ have finite overlap. Using $\|g\|_{p}=\rho^{-\frac{1}{p}}\|f\|_{p}$ and juggling some indices, we obtain

$$
(74) \lesssim \rho^{\frac{3}{2}-\frac{3+2 p_{0}}{2 p}} \alpha^{1-\frac{3+p_{0}}{2 p}} \delta^{\frac{p_{0}}{p}-1} .
$$

The power of $\alpha$ is negative by (76), and $\alpha \gtrsim \delta$ so by summing over $\alpha$ we bound the left side of $(73)$ by $\rho^{\frac{3}{2}-\frac{3+2 p_{0}}{2 p}} \delta^{\frac{p_{0}-3}{2 p}}$. The power of $\rho$ here is positive if $p$ is close to $\frac{17}{6}$, so we obtain

$$
\text { left hand side of }(73) \lesssim \delta^{\frac{p_{0}-3}{2 p}}\|f\|_{p} \text {. }
$$

Now we are done (with case (i) of (61)), since $\frac{3-p_{0}}{2 p}<\frac{3}{p}-1$ by (76).

Case (ii) is simply a scaling argument. We consider the same map $T\left(x_{1}, x_{2}, x_{3}\right)=\left(x_{1}, x_{2}, \rho x_{3}\right)$, where now $\rho \geq 1$. If $e \in \Omega_{1}$ and $t$ is a $\delta$ neighborhood of a line in the $e_{T}$ direction, then it is not difficult to see that $T^{-1} t$ is essentially a $\delta$-neighborhood of a line in the $e$ direction. Consequently, since volumes are distorted by $\rho$,

$$
\bar{F}_{\delta}\left(e_{T}\right) \lesssim \rho \overline{(F \circ T)}_{\delta}(e) .
$$

For large $\rho$ the map $e \rightarrow e_{T}$ contracts areas by a factor of roughly $\rho^{2}$, so

$$
\begin{aligned}
\left\|\bar{F}_{\delta}\right\|_{L^{q}\left(\Omega_{\rho}\right)} & =\rho^{1-\frac{2}{q}}\|\overline{(F \circ T)} \delta\|_{L^{q}\left(\Omega_{1}\right)} \\
& \lesssim \rho^{1-\frac{2}{q}} \delta^{-\left(\frac{3}{p}-1\right)+\epsilon}\|F \circ T\|_{p} \\
& =\rho^{1-\frac{2}{q}-\frac{1}{p}} \delta^{-\left(\frac{3}{p}-1\right)+\epsilon}\|F\|_{p} \\
& =\delta^{-\left(\frac{3}{p}-1\right)+\epsilon}\|F\|_{p}
\end{aligned}
$$

where we used the $\rho=1$ case and the relation between $p$ and $q$. This finishes the proof of (61), hence of Lemma 5.3.

Proof of Theorem $3^{\prime}$. We may assume that $p$ is sufficiently close to $\frac{17}{6}$. We claim first that if $\operatorname{supp} f \subset\left\{(s, t): \frac{r}{2} \leq s \leq 2 r\right\}$ then

$$
\left\|\bar{F}_{\delta}\right\|_{L^{q}(\tilde{\Omega})} \lesssim r^{-\epsilon} \delta^{-\left(\frac{3}{p}-1\right)+\epsilon}\|F\|_{p}
$$

with $\epsilon$ as in Lemma 5.3.

The case $r=1$ is the content of Lemma 5.3. The general case will follows by scaling. If we set $G(x)=F(r x)$ then we have

$$
\bar{F}_{\delta}(e)=r \bar{G}_{\frac{\delta}{r}}(e)
$$

so by applying Lemma 5.3 to $G$ we get

$$
\left\|\bar{F}_{\delta}\right\|_{L^{q}(\tilde{\Omega})}=r\left\|\bar{G}_{\delta}\right\|_{L^{q}(\tilde{\Omega})}
$$




$$
\begin{aligned}
& \lesssim r\left(\frac{\delta}{r}\right)^{-\left(\frac{3}{p}-1\right)+\epsilon}\|G\|_{p} \\
& =r\left(\frac{\delta}{r}\right)^{-\left(\frac{3}{p}-1\right)+\epsilon} r^{\frac{3}{p}}\|F\|_{p},
\end{aligned}
$$

which is (78).

An easy fact about $F_{\delta}^{*}$ is that $F_{\delta}^{*}(e) \lesssim F_{\delta}^{*}(\tilde{e})$ if $|e-\tilde{e}| \leq \delta$. Consequently $\left\|F_{\delta}^{*}\right\|_{q}$ and $\left\|F_{\delta}^{*}\right\|_{L^{q}(\tilde{\Omega})}$ are comparable, so (78) implies that

$$
\left\|F_{\delta}^{*}\right\|_{q} \lesssim r^{-\epsilon} \delta^{-\left(\frac{3}{p}-1\right)+\epsilon}\|F\|_{p}, \quad \operatorname{supp} f \subset\left\{(s, t): \frac{r}{2} \leq s \leq 2 r\right\} .
$$

It follows on summing a geometric series that

$$
\left\|F_{\delta}^{*}\right\|_{q} \lesssim \delta^{-\left(\frac{3}{p}-1\right)}\|F\|_{p}
$$

provided $\operatorname{supp} f \subset\{(s, t): s \geq \delta\}$.

We still have to prove estimate (79) when $\operatorname{supp} f \subset\{(s, t): s \leq \delta\}$. In order to do so we first consider a function $\psi: \mathbb{R} \rightarrow \mathbb{R}$, and define

$$
\mathcal{A} \psi(t)=\sup _{I} \frac{1}{|I|} \int_{I}|\psi|
$$

where $I$ runs over all intervals of length $t$. We can dominate $\mathcal{A} \psi(t)$ by the average over $(0, t)$ of the nonincreasing rearrangement of $|\psi|$ so by Hardy's inequality

$$
\int_{0}^{\infty} t^{\frac{q}{p}-1} \mathcal{A} \psi(t)^{q} d t \lesssim\|\psi\|_{p}^{q}, \quad 1<p \leq q<\infty .
$$

Define a function $\psi$ of one variable via $\psi(x)=\delta^{-2} \int F\left(x_{1}, x_{2}, x\right) d x_{1} d x_{2}$. Because of the support restriction on $F$, it is easy to see that

$$
F_{\delta}^{*}(e) \lesssim \frac{\delta}{\theta} \mathcal{A} \psi\left(\frac{\delta}{\theta}\right),
$$

where $\theta$ is the angle between $e$ and the $x_{3}$ axis. Consequently,

$$
\begin{aligned}
\left\|F_{\delta}^{*}\right\|_{q}^{q} & \lesssim \int_{0}^{\frac{\pi}{2}}\left(\frac{\delta}{\theta} \mathcal{A} \psi\left(\frac{\delta}{\theta}\right)\right)^{q} \theta d \theta \\
& \lesssim \int_{0}^{\infty}(t \mathcal{A} \psi(t))^{q} \delta^{2} t^{-3} d t \\
& \lesssim \delta^{2}\|\psi\|_{p}^{q}
\end{aligned}
$$

since $q-3=\frac{q}{p}-1$.

On the other hand, it follows using Hölder's inequality that $\|\psi\|_{p} \lesssim$ $\delta^{-\frac{2}{p}}\|F\|_{p}$. We conclude that $\left\|F_{\delta}^{*}\right\|_{q} \lesssim \delta^{\frac{2}{q}-\frac{2}{p}}\|F\|_{p}=\delta^{-\left(\frac{3}{p}-1\right)}\|F\|_{p}$ and the proof of Theorem $3^{\prime}$ is complete. 


\section{References}

[1] A.S. Besicovitch and R. Rado, A plane set of measure zero containing circumferences of every radius, J. London Math. Soc., 43 (1968), 717-719.

[2] J. Bourgain, Besicovitch type maximal operators and applications to Fourier analysis, Geometric and Functional Analysis, 1 (1990), 147-187.

[3] A. Cordoba, The Kakeya maximal function and spherical summation multipliers, Amer. J. Math., 99 (1977), 1-22.

[4] R.L. Graham, B.L. Rothschild and J. H. Spencer, Ramsey Theory, 2nd edition, WileyInterscience, 1990.

[5] J.R. Kinney, A thin set of circles, Amer. Math. Monthly, 75 (1968), 1077-1081.

[6] J.E. Marsden, Elementary Classical Analysis, W.H. Freeman, 1974.

[7] J.M. Marstrand, Packing circles in the plane, Proc. London Math. Soc., 55 (1987), $37-58$.

[8] G. Mockenhoupt, A. Seeger and C. Sogge, Wave front sets and Bourgain's circular maximal theorem, Ann. Math., 134 (1992), 207-218.

[9] H. Pecher, Nonlinear small data scattering for the wave and Klein-Gordon equation, Math. Z., 185 (1984), 261-270.

[10] C. Sogge, Propagation of singularities and maximal functions in the plane, Inv. Math., 104 (1991), 349-376.

[11] _ Fourier Integrals in Classical Analysis, Cambridge University Press, 1993.

[12] M. Talagrand, Sur la measure de la projection d'un compact et certaines familles de cercles, Bull. Sci. Math., 104(2) (1980), 225-231.

[13] T. Wolff, An improved bound for Kakeya type maximal functions, Revista Math. Iberoamericana, 11 (1995), 651-674.

Received January 22, 1996 and revised May 20, 1997. The second author was supported by DMS 93-07872.

Ryerson Polytechnic University

Toronto, Ontario M5B 2K3

CANADA

E-mail address: lkolasa@acs.ryerson.ca

253-37 CALTECH

Pasadena, CA 91125

E-mail address: wolff@cco.caltech.edu 